\title{
Discrete gauge groups in F-theory models on genus-one fibered Calabi-Yau 4-folds without section
}

\author{
Yusuke Kimura \\ Yukawa Institute for Theoretical Physics, Kyoto University, \\ Kitashirakawa Oiwakecho, Sakyo-ku, Kyoto 606-8502, Japan \\ E-mail: kimura@yukawa.kyoto-u.ac.jp
}

ABSTRACT: We determine the discrete gauge symmetries that arise in F-theory compactifications on examples of genus-one fibered Calabi-Yau 4-folds without a section. We construct genus-one fibered Calabi-Yau 4-folds using Fano manifolds, cyclic 3-fold covers of Fano 4 -folds, and Segre embeddings of products of projective spaces. Discrete $\mathbb{Z}_{5}, \mathbb{Z}_{4}, \mathbb{Z}_{3}$ and $\mathbb{Z}_{2}$ symmetries arise in these constructions. We introduce a general method to obtain multisections for several constructions of genus-one fibered Calabi-Yau manifolds. The pullbacks of hyperplane classes under certain projections represent multisections to these genus-one fibrations. We determine the degrees of these multisections by computing the intersection numbers with fiber classes. As a result, we deduce the discrete gauge symmetries that arise in F-theory compactifications. This method applies to various Calabi-Yau genus-one fibrations.

Keywords: Differential and Algebraic Geometry, F-Theory, Gauge Symmetry, Superstring Vacua

ArXiv EPrint: 1608.07219 


\section{Contents}

\section{Introduction $\quad 2$}

2 Constructions of genus-one fibered Calabi-Yau 4-folds 4

2.1 Intersections of two hyperplane classes in product of del Pezzo 3-folds 4

2.2 Cyclic 3-fold covers of $\mathbb{P}^{1}$ times del Pezzo 3-folds 7

2.3 Constructions involving Segre embeddings of $\mathbb{P}^{2} \times \mathbb{P}^{2}$ and $\mathbb{P}^{1} \times \mathbb{P}^{1} \times \mathbb{P}^{1} \quad 7$

2.4 Hypersurfaces in $\mathbb{P}^{2}$ times Fano 3-folds 8

2.5 Complete intersections in $\mathbb{P}^{3}$ times Fano 3-folds 8

$\begin{array}{lll}2.6 & \text { Double covers of } \mathbb{P}^{1} \text { times Fano 3-folds } & 10\end{array}$

3 Discrete gauge symmetry

3.1 Discrete $\mathbb{Z}_{2}, \mathbb{Z}_{3}, \mathbb{Z}_{4}$ and $\mathbb{Z}_{5}$ symmetries on intersections of two hyperplane classes in product of del Pezzo 3-folds

3.2 Discrete $\mathbb{Z}_{3}$ symmetry on cyclic 3 -fold covers of $\mathbb{P}^{1}$ times del Pezzo 3-folds

3.3 Discrete $\mathbb{Z}_{3}$ and $\mathbb{Z}_{2}$ symmetries on constructions involving Segre embeddings of $\mathbb{P}^{2} \times \mathbb{P}^{2}$ and $\mathbb{P}^{1} \times \mathbb{P}^{1} \times \mathbb{P}^{1}$

3.3.1 $\mathbb{Z}_{3}$ symmetry in constructions involving Segre embeddings of $\mathbb{P}^{2} \times \mathbb{P}^{2}$ into $\mathbb{P}^{8}$

3.3.2 $\mathbb{Z}_{2}$ symmetry in constructions involving Segre embeddings of $\mathbb{P}^{1} \times$ $\mathbb{P}^{1} \times \mathbb{P}^{1}$ into $\mathbb{P}^{7}$

3.4 Discrete $\mathbb{Z}_{3}$ symmetry on hypersurfaces in $\mathbb{P}^{2}$ times Fano 3-folds

3.5 Discrete $\mathbb{Z}_{4}, \mathbb{Z}_{2}$ symmetries on complete intersections in $\mathbb{P}^{3}$ times Fano 3-folds 12

3.6 Discrete $\mathbb{Z}_{2}$ symmetry on double covers of $\mathbb{P}^{1}$ times Fano 3 -folds

4 Conclusion

A Gauge theories and matter spectra on $(2,1,1,1)$ and $(2,1,1,1)$ complete intersections in $\mathbb{P}^{3} \times \mathbb{P}^{1} \times \mathbb{P}^{1} \times \mathbb{P}^{1}$

A.1 Forms of the discriminant components, non-Abelian gauge groups on 7branes and Jacobian fibration

$\begin{array}{lll}\text { A.2 Mordell-Weil group of the Jacobian fibration } & 18\end{array}$

A.3 Euler characteristic and condition on 4-form flux to cancel the tadpole 19

A.4 Potential matter fields and Yukawa couplings 20 


\section{Introduction}

F-theory [1-3] is an extension of type IIB superstring theory, which provides a nonperturbative formulation. F-theory is compactified on genus-one fibered Calabi-Yau $m$-folds (with or without a section) where the axio-dilaton, $\tau=C_{0}+i e^{-\phi}$, is identified with the modular parameter of a genus-one fiber, enabling the axio-dilaton to have $S L_{2}(\mathbb{Z})$ monodromy. 7-branes are magnetic sources for the RR 0 -form $C_{0}$, and 7-branes are wrapped on the components of a discriminant locus, i.e., the codimension 1 locus in the base over which fibers degenerate.

Until recently, in most literature, elliptically fibered Calabi-Yau $m$-folds that admit a global section were used as compactification geometries for F-theory. (For models with a global section, see, for example, [4-21].) However, a Calabi-Yau $m$-fold with a torus fibration does not typically admit a section. Therefore, it is possible to consider genus-one fibered Calabi-Yau $m$-folds lacking a global section as compactification geometries for Ftheory. In recent years, such F-theory compactifications on genus-one fibrations without a section have been considered in several studies, initiated in $[22,23] .{ }^{1}$ For recent progress in F-theory compactifications on genus-one fibrations without a section, see also, for example, [26-36]. In [23], Morrison and Taylor considered the Jacobian of a genus-one fibration without a section to show that the F-theory geometry without a section fits into the moduli of Weierstrass models. They observed that in the moduli of Weierstrass models, the geometry of genus-one fibrations includes the geometry of elliptic fibrations with a section. They argued that moving from an F-theory model with a global section to an F-theory model on genus-one fibrations without a section in the moduli space can be viewed as a Higgsing process, in which a $\mathrm{U}(1)$ gauge symmetry is broken and a discrete gauge symmetry ${ }^{2}$ remains.

The Calabi-Yau genus-one fibrations with an identical Jacobian fibration, $J$, form a group referred to as the Tate-Shafarevich group, $\amalg(J)$. A genus-one fibration $Y$ and the Jacobian fibration $J(Y)$ have identical $\tau$ functions. Therefore, F-theory compactifications on distinct genus-one fibrations in $\amalg(J)$ describe physically equivalent theories. As discussed in [23], the Tate-Shafarevich group $\amalg(J(Y)$ ) (of the Jacobian $J(Y)$ ) is identified with the discrete gauge group that forms in F-theory compactification on a Calabi-Yau genus-one fibration $Y$ [25]. Concretely, in F-theory compactified on a genus-one fibration with an $n$-section, a discrete $\mathbb{Z}_{n}$ symmetry arises as the remnant of a broken $\mathrm{U}(1)^{n-1}$. For example, a discrete $\mathbb{Z}_{2}$ gauge symmetry arises in F-theory compactification on a genus-one fibration with a bisection, and when a genus-one fibration has a 3-section, a discrete $\mathbb{Z}_{3}$ symmetry arises in F-theory compactification.

In this note, we determine the discrete gauge symmetries that arise in F-theory compactifications on several constructions of genus-one fibered Calabi-Yau 4-folds without a section, by specifying the degrees of multisections. We build genus-one fibered Calabi-Yau manifolds that lack a global section using Fano manifolds. Fano manifolds are a generalization of (products of) projective spaces. We consider the following constructions of genus-one fibered Calabi-Yau 4-folds:

\footnotetext{
${ }^{1}$ F-theory models without a section were considered in [24, 25].

${ }^{2}$ For recent progress in discrete gauge symmetries, see, for example, [37-53].
} 
1. Intersection of two hyperplane classes in the product of two Fano 3-folds.

2. Cyclic 3-fold covers of $\mathbb{P}^{1}$ times Fano 3-folds.

3. Constructions involving Segre embeddings of $\mathbb{P}^{2} \times \mathbb{P}^{2}$ and $\mathbb{P}^{1} \times \mathbb{P}^{1} \times \mathbb{P}^{1}$.

4. Hypersurfaces in $\mathbb{P}^{2}$ times Fano 3-folds.

5. Complete intersections in $\mathbb{P}^{3}$ times Fano 3-folds.

6. Double covers of $\mathbb{P}^{1}$ times Fano 3-folds.

In the six constructions $1 . \sim 6$., we particularly use Fano 3 -folds of index $2, V_{d}$ $(d=2, \cdots, 8)$. These Fano 3 -folds $V_{d}$ were discussed in [54-56]. In this note, Fano 3 -folds $V_{d}$ are referred to as del Pezzo 3-folds of degree d, following $[55,56]$. We do not consider the del Pezzo 3-fold of degree 1, $V_{1}$, in this note.

We determine the discrete gauge symmetries that form in F-theory compactifications on the above six constructions of genus-one fibered Calabi-Yau 4-folds. In this note, we introduce a general method which is applicable to all of the above six constructions of Calabi-Yau genus-one fibrations.

As stated above, a discrete $\mathbb{Z}_{n}$ gauge symmetry forms in F-theory compactification on a Calabi-Yau genus-one fibration with a multisection of degree $n$. We introduce a general method to determine the degrees of multisections, as follows: in each of the six CalabiYau constructions introduced above, genus-one fibers are embedded in a Fano manifold. The pullback of a hyperplane class in this Fano manifold to a Calabi-Yau space gives a multisection. The pullback of a point class in the base 3-fold, on the other hand, represents the fiber class. The degree of a multisection represents the number of intersection points with a fiber. (Therefore, a multisection of degree 1, which intersects with a fiber in a single point, is a global section.) Said differently, the intersection number of a multisection with the fiber class gives the degree of a multisection. Representing a multisection as the pullback of a hyperplane class enables to compute the intersection number with the fiber class. This method applies to all of the six Calabi-Yau constructions 1. 6. . Using this method, we deduce the discrete gauge groups that arise in F-theory compactified on the six constructions of Calabi-Yau 4-folds.

This method is considerably general, and is also applicable to similar constructions of genus-one fibered Calabi-Yau manifolds. This method might be useful in determining discrete gauge symmetries in F-theory approach.

The constructions 1.,2. and 3. provide novel examples of F-theory compactifications in which discrete symmetries form. In construction 1 ., discrete $\mathbb{Z}_{2}, \mathbb{Z}_{3}, \mathbb{Z}_{4}$ and $\mathbb{Z}_{5}$ gauge symmetries arise in F-theory compactifications employing del Pezzo 3 -folds $V_{2}, V_{3}, V_{4}$ and $V_{5}$, respectively. In construction 2 , a discrete $\mathbb{Z}_{3}$ symmetry arises in F-theory compactifications. In construction 3 , a discrete $\mathbb{Z}_{3}$ gauge symmetry arises in F-theory compactifications involving Segre embedding of $\mathbb{P}^{2} \times \mathbb{P}^{2}$ into $\mathbb{P}^{8}$. A discrete $\mathbb{Z}_{2}$ symmetry arises in F-theory compactifications involving Segre embedding of $\mathbb{P}^{1} \times \mathbb{P}^{1} \times \mathbb{P}^{1}$ into $\mathbb{P}^{7}$.

In construction 4., we consider hypersurfaces whose genus-one fibers are cubic hypersurfaces in $\mathbb{P}^{2}$. F-theory compactifications on Calabi-Yau genus-one fibrations whose 
fibers are cubic hypersurfaces in $\mathbb{P}^{2}$ were considered in $[27,31,34,36]$. In such F-theory compactifications, a discrete $\mathbb{Z}_{3}$ symmetry arises $[27,31]$.

In construction 5., we consider the complete intersections whose genus-one fibers are the complete intersections of two quadrics in $\mathbb{P}^{3}$. F-theory compactifications on Calabi-Yau genus-one fibrations, whose fibers are complete intersections of two quadric hypersurfaces in $\mathbb{P}^{3}$ were studied in $[35,50]$. In these F-theory compactifications, a discrete $\mathbb{Z}_{4}$ gauge symmetry arises [50].

In construction 6., we consider Calabi-Yau spaces as double covers, whose genus-one fibers are double covers of $\mathbb{P}^{1}$ ramified over 4 points. F-theory compactifications of this kind were considered in $[23,35,36]$. In F-theory compactifications on these double covers, a discrete $\mathbb{Z}_{2}$ symmetry arises [23].

This paper is structured as follows: in section 2, we introduce the six constructions of genus-one fibered Calabi-Yau 4-folds that lack a global section. In section 3, we deduce discrete symmetries that form in F-theory compactifications on the six Calabi-Yau constructions. We represent multisections as pullbacks of hyperplane classes, and we compute the intersection numbers of these pullbacks of hyperplane classes with the fiber class to obtain the discrete symmetries. Constructions 1., 2. and 3. give novel examples of F-theory compactifications in which discrete symmetries form. In section 4, we state the concluding remarks. The continuous gauge theories and matter spectra in F-theory compactification on the special complete intersections whose K3 fibers are isomorphic to the Fermat quartic will be discussed in appendix A.

\section{Constructions of genus-one fibered Calabi-Yau 4-folds}

In this section, we introduce constructions of genus-one fibrations without a section that are Calabi-Yau 4-folds. In section 3, we determine the discrete gauge symmetries that arise in F-theory compactifications on these genus-one fibrations.

\subsection{Intersections of two hyperplane classes in product of del Pezzo 3-folds}

In this section, we consider special Fano 3-folds, called the del Pezzo 3-folds of degree $d$, $V_{d}, d=2, \cdots, 8$, to construct genus-one fibered Calabi-Yau 4-folds. Structures of the del Pezzo 3 -folds $V_{d}, d=2, \cdots, 8$ [54-56] are displayed in table 1 below.

We consider the product of two del Pezzo 3 -folds $V_{D} \times V_{E}$, and we consider the intersection of two hyperplane sections ${ }^{3} \mathcal{O}_{V_{D} \times V_{E}}(1,1)$ in the product $V_{D} \times V_{E}$. The resulting 4-fold $Y_{4}$ has the trivial canonical bundle; therefore it is a Calabi-Yau 4-fold.

By construction, Calabi-Yau 4-fold $Y_{4}$ has projection $p$ onto $V_{E}$, and projection $q$ onto $V_{D}$. Fiber of projection $p$ is the intersection of two hyperplane classes $\mathcal{O}_{V_{D}}(1)$ in del Pezzo 3 -fold $V_{D}$, therefore it is a genus-one curve. Similarly, fiber of projection $q$ is the intersection of two hyperplane classes $\mathcal{O}_{V_{E}}(1)$ in del Pezzo 3 -fold $V_{E}$, i.e. a genus-one curve. Therefore, projections $p, q$ are genus-one fibrations.

\footnotetext{
${ }^{3}$ Del Pezzo 3-fold $V_{8}=\mathbb{P}^{3}$ is seen as a subvariety embedded in $\mathbb{P}^{9}$ using the Veronese map of degree $2, v_{2}$ : $V_{8}=\mathbb{P}^{3} \hookrightarrow \mathbb{P}^{9}$. Hyperplane class $\mathcal{O}_{V_{8}}(1)$ in del Pezzo 3 -fold $V_{8}$ is defined as the restriction of hyperplane class $\mathcal{O}_{\mathbb{P}^{9}}(1)$ in $\mathbb{P}^{9}$ to $V_{8}: \mathcal{O}_{V_{8}}(1):=\left.\mathcal{O}_{\mathbb{P}^{9}}(1)\right|_{V_{8}}$. Therefore, $\mathcal{O}_{V_{8}}(1)=v_{2}^{*} \mathcal{O}_{\mathbb{P}^{9}}(1)=\mathcal{O}_{\mathbb{P}^{3}}(2)$.
} 


\begin{tabular}{|c|c|}
\hline Degree $d$ of del Pezzo 3-fold $V_{d}$ & Structure \\
\hline 2 & Double cover of $\mathbb{P}^{3}$ \\
3 & Cubic 3-fold in $\mathbb{P}^{4}$ \\
4 & Intersection of three hyperplane sections in $G r(2,5) \subset \mathbb{P}^{9}$ \\
5 & $\mathbb{P}^{1} \times \mathbb{P}^{1} \times \mathbb{P}^{1}$ \\
6 & Blow-up of $\mathbb{P}^{3}$ at a point \\
7 & $\mathbb{P}^{3}$ \\
\hline
\end{tabular}

Table 1. List of del Pezzo 3-folds with degree $d, d=2, \cdots, 8[54-56]$. Gr $(2,5)$ denotes the complex Grassmannian of two-dimensional linear subspaces in $\mathbb{C}^{5} . \operatorname{Gr}(2,5)$ is embedded inside $\mathbb{P}^{9}$ using the Plücker embedding; the restriction of a hyperplane section in $\mathbb{P}^{9}$ to $G r(2,5)$ gives a hyperplane section of $\operatorname{Gr}(2,5)$. The del Pezzo 3-fold of degree $8 V_{8}$ is the image of $\mathbb{P}^{3}$ embedded in $\mathbb{P}^{9}$ under the Veronese embedding.

In this note, we focus on the case $D=2,3,4,5$ and $E=2, \cdots, 8$, and we choose $V_{E}$ to be the base 3 -fold. Genus-one fiber is the intersection of two hyperplanes $\mathcal{O}_{V_{D}}(1)$ in del Pezzo 3-fold $V_{D}, D=2,3,4,5$.

In section 3.1 , we deduce that the pullback of a hyperplane class in $V_{D}$

$$
q^{*} \mathcal{O}_{V_{D}}(1)
$$

gives a multisection of degree $D$ to the fibration. Therefore, we find that a discrete $\mathbb{Z}_{D}$ gauge symmetry $(D=2,3,4,5)$ forms in F-theory compactification on Calabi-Yau 4-fold $Y_{4}$.

We note that $\mathbb{P}^{1} \times \mathbb{P}^{2}$ may be used as the base 3 -fold, in place of $V_{E}$. For this case, Calabi-Yau 4-fold is built as the complete intersection of $\mathcal{O}_{V_{D} \times \mathbb{P}^{1} \times \mathbb{P}^{2}}(1,1,2)$ and $\mathcal{O}_{V_{D} \times \mathbb{P}^{1} \times \mathbb{P}^{2}}(1,1,1)$ hypersurfaces in $V_{D} \times \mathbb{P}^{1} \times \mathbb{P}^{2}$. Natural projection onto $\mathbb{P}^{1} \times \mathbb{P}^{2}$ gives a genus-one fibration, and the pullback (2.1) gives a multisection of degree $D$, same as the case stated above, in which the base 3 -fold is $V_{E}$.

We show that a generic member of genus-one fibered Calabi-Yau 4-folds $Y_{4}$, constructed as complete intersections of two $\mathcal{O}_{V_{D} \times V_{E}}(1,1)$ classes in $V_{D} \times V_{E}(D=2,3, \cdots, 6,8, E=$ $2, \cdots, 8$ ), does not have a global section. (The base 3 -fold of genus-one fibration is $V_{E}$.) To show that $Y_{4}$ lacks a global section, we prove that the Picard group $\operatorname{Pic}\left(Y_{4}\right)$, which is isomorphic to the group of divisor classes in $Y_{4}$, is generated by the pullbacks of the divisors in $V_{D}$ and the pullbacks of the divisors in $V_{E}$. First, we assume that $D \neq 6,8, E \neq 6,7$. The cases where $D=6,8$ or $E=6,7$ are merely special cases, and similar arguments as that stated below apply to these special cases. Since

$$
H^{1}\left(V_{D}\right)=0, \quad H^{1}\left(V_{E}\right)=0,
$$

(these follow from the fact that $V_{D}$ and $V_{E}$ are Fano 3-folds), by Künneth formula, we find that

$$
H^{2}\left(V_{D} \times V_{E}, \mathbb{Z}\right) \cong \mathbb{Z}^{2}
$$


By Lefschetz theorem,

$$
H^{2}\left(Y_{4}, \mathbb{Z}\right) \cong H^{2}\left(V_{D} \times V_{E}, \mathbb{Z}\right) \cong \mathbb{Z}^{2}
$$

Since $V_{D}$ and $V_{E}$ are Fano 3 -folds, $H^{1}\left(V_{D} \times V_{E}, \mathbb{Z}\right)=0$, therefore by Lefschetz theorem $H^{1}\left(Y_{4}, \mathbb{Z}\right)=0$. This shows that $\operatorname{Pic}^{0}\left(Y_{4}\right)=0$, and thus $\operatorname{Pic}\left(Y_{4}\right) \cong N S\left(Y_{4}\right)$. By the Lefschetz $(1,1)$ theorem $N S\left(Y_{4}\right) \cong H^{2}\left(Y_{4}, \mathbb{Z}\right) \cap H^{1,1}\left(Y_{4}\right)$, therefore the rank of the Picard group is bounded above by the rank of $H^{2}\left(Y_{4}, \mathbb{Z}\right)$, which is 2 by (2.4). The pullbacks of $\mathcal{O}(1)$ classes in $V_{D}$ and $V_{E}$ give divisors of $Y_{4}$, thus we conclude that these pullbacks generate the Picard group $\operatorname{Pic}\left(Y_{4}\right)$. The pullback $p^{*} \mathcal{O}_{V_{E}}(1)$ is the pullback of a divisor in the base $V_{E}$; therefore, the pullback $p^{*} \mathcal{O}_{V_{E}}(1)$ is parallel to the fiber class $F$, namely the pullback $p^{*} \mathcal{O}_{V_{E}}(1)$ has an intersection number 0 with the fiber class $F$. On the other hand, as we will see in section 3.1, the pullback $q^{*} \mathcal{O}_{V_{D}}(1)$ of $\mathcal{O}_{V_{D}}(1)$ class in $V_{D}$ gives a $D$-section, when $D=2,3,4,5$. Therefore, a divisor in $Y_{4}$, which is $n q^{*} \mathcal{O}_{V_{D}}(1)+m p^{*} \mathcal{O}_{V_{E}}(1)$ for some integers $n, m$, has an intersection number a multiple of $D$ with the fiber class $F$. $\left(\left(n q^{*} \mathcal{O}_{V_{D}}(1)+m p^{*} \mathcal{O}_{V_{E}}(1)\right) \cdot F=n D\right.$. $)$ This shows that $Y_{4}$ has only multisections whose degrees are multiples of $D$, thus we conclude that $Y_{4}$ does not have a global section.

When $D \neq 6,8, E=6$, the second Betti number of the del Pezzo 3-fold of degree $6 V_{6}$ is $b_{2}\left(V_{6}\right)=3$, therefore we have

$$
H^{2}\left(V_{D} \times V_{E}, \mathbb{Z}\right) \cong \mathbb{Z}^{4}
$$

By Lefschetz theorem, we obtain

$$
H^{2}\left(Y_{4}, \mathbb{Z}\right) \cong \mathbb{Z}^{4}
$$

For this case, the pullback $p^{*} \mathcal{O}_{V_{6}}(1)$ splits into three classes: $p^{*} \mathcal{O}_{\mathbb{P}^{1} \times \mathbb{P}^{1} \times \mathbb{P}^{1}}(1,0,0)$, $p^{*} \mathcal{O}_{\mathbb{P}^{1} \times \mathbb{P}^{1} \times \mathbb{P}^{1}}(0,1,0)$ and $p^{*} \mathcal{O}_{\mathbb{P}^{1} \times \mathbb{P}^{1} \times \mathbb{P}^{1}}(0,0,1)$. This comes from the fact that $V_{6}$ is the direct product of three projective lines. Therefore, $Y_{4}$ has four divisors: these three pullbacks, and the pullback $q^{*} \mathcal{O}_{V_{D}}(1)$. From (2.6), we find that the rank of the Picard group $\operatorname{Pic}\left(Y_{4}\right)$ is bounded above by 4 . Thus, we conclude that the four divisors that we found generate the Picard group $\operatorname{Pic}\left(Y_{4}\right)$. Three pullbacks $p^{*} \mathcal{O}_{\mathbb{P}^{1} \times \mathbb{P}^{1} \times \mathbb{P}^{1}}(1,0,0), p^{*} \mathcal{O}_{\mathbb{P}^{1} \times \mathbb{P}^{1} \times \mathbb{P}^{1}}(0,1,0)$ and $p^{*} \mathcal{O}_{\mathbb{P}^{1} \times \mathbb{P}^{1} \times \mathbb{P}^{1}}(0,0,1)$ are parallel to the fiber class $F$, and the pullback $q^{*} \mathcal{O}_{V_{D}}(1)$ has an intersection number $D$ with the fiber class $F$. Thus, every divisor in $Y_{4}$ has an intersection number a multiple of $D$ with the fiber class $F$. This shows that $Y_{4}$ does not have a global section.

When $D \neq 6,8, E=7$, the base 3 -fold $V_{7}$ is blow-up of $\mathbb{P}^{3}$ at a point; therefore, the Picard group of $V_{7} \operatorname{Pic}\left(V_{7}\right)$ is generated by $\mathcal{O}_{V_{7}}(1)$ and the exceptional divisor E. Since $b_{2}\left(V_{7}\right)=2$,

$$
H^{2}\left(V_{D} \times V_{7}, \mathbb{Z}\right) \cong \mathbb{Z}^{3}
$$

and by Lefschetz theorem

$$
H^{2}\left(Y_{4}, \mathbb{Z}\right) \cong \mathbb{Z}^{3}
$$

Thus, the rank of the Picard group $\operatorname{Pic}\left(Y_{4}\right)$ is bounded by 3 . The pullbacks $p^{*} \mathcal{O}_{V_{7}}(1), p^{*} E$ and $q^{*} \mathcal{O}_{V_{D}}(1)$ give divisors of $Y_{4}$, therefore we conclude that these pullbacks generate the Picard group Pic $\left(Y_{4}\right) \cdot p^{*} \mathcal{O}_{V_{7}}(1)$ and $p^{*} E$ are pullbacks of divisors in the base 3 -fold $V_{7}$, thus 
they are parallel to the fiber class $F$. It follows that any divisor in $Y_{4}$ has an intersection number a multiple of $D$ with the fiber class $F$. This shows that $Y_{4}$ does not have a section.

When $D=6$, multisection $q^{*} \mathcal{O}_{V_{6}}(1)$ splits into three bisections. (We discuss this case from the perspective of Segre embeddings in section 2.3 and 3.3.2.) When $D=8$, multisection $q^{*} \mathcal{O}_{V_{8}}(1)$ represents a 4 -section. Similar arguments as that stated above show that for these cases $Y_{4}$ does not have a global section. When $D=7, Y_{4}$ admits a global section.

\subsection{Cyclic 3-fold covers of $\mathbb{P}^{1}$ times del Pezzo 3 -folds}

Cyclic 3-fold covers of $\mathbb{P}^{1} \times V_{E}$ ramified along a $\mathcal{O}_{\mathbb{P}^{1} \times V_{E}}(3,3) 3$-fold are Calabi-Yau 4-folds. As in section 2.1, $V_{E}$ denotes the del Pezzo 3-fold of degree $E$, with $E=2, \cdots, 8$.

By construction, a cyclic 3-fold cover, $Y$, has projection $p$ onto $V_{E}$, and projection $q$ onto $\mathbb{P}^{1}$. Fiber of projection $p$ onto $V_{E}$ is a cyclic 3 -fold cover of a rational curve $\mathbb{P}^{1}$ ramified over 3 points, which is a genus-one curve. Therefore, projection $p$ gives a genusone fibration.

In section 3.2 , we will find that the pullback of a hyperplane class in $\mathbb{P}^{1}$

$$
q^{*} \mathcal{O}_{\mathbb{P}^{1}}(1)
$$

gives a 3 -section to the fibration. Thus, a discrete $\mathbb{Z}_{3}$ gauge symmetry arises in F-theory compactification on cyclic 3-fold cover $Y$.

We remark that the possible non-Abelian gauge symmetries on the 7-branes in F-theory compactifications on cyclic 3 -fold covers of $\mathbb{P}^{1} \times V_{E}$ are highly constrained by the symmetry. Similar observations are made in $[34,36]$. Genus-one fiber has the automorphism group of order 3, therefore every smooth fiber is the Fermat curve. Indeed, a rational curve $\mathbb{P}^{1}$ with 3 fixed points has the constant moduli, therefore the complex structure of its cyclic 3 -fold cover is unique. It follows that fibers have the j-invariant 0 throughout the base; as a result, singular fibers have the j-invariant 0 . Thus, the possible fiber types ${ }^{4}$ are $I I, I V$, $I_{0}^{*}, I V^{*}$ and $I I^{*}$; the possible non-Abelian gauge symmetries that can arise on the 7-branes are: $\mathrm{SU}(3), \mathrm{SO}(8), E_{6}$ and $E_{8}$.

\subsection{Constructions involving Segre embeddings of $\mathbb{P}^{2} \times \mathbb{P}^{2}$ and $\mathbb{P}^{1} \times \mathbb{P}^{1} \times \mathbb{P}^{1}$}

We construct Calabi-Yau 4-folds using Segre embeddings of products of projective spaces. We consider the following two Segre embeddings:

$$
\begin{array}{r}
\mathbb{P}^{2} \times \mathbb{P}^{2} \hookrightarrow \mathbb{P}^{8} \\
\mathbb{P}^{1} \times \mathbb{P}^{1} \times \mathbb{P}^{1} \hookrightarrow \mathbb{P}^{7}
\end{array}
$$

We denote a quadric hypersurface in $\mathbb{P}^{4}$ by $Q_{3}$. We consider the direct product of $Q_{3}$, and $\mathbb{P}^{2} \times \mathbb{P}^{2}$ embedded in $\mathbb{P}^{8}$ under the Segre embedding: $Q_{3} \times \mathbb{P}^{2} \times \mathbb{P}^{2}$. This is a Fano 7-fold, and the intersection of three hyperplane sections $\mathcal{O}_{Q_{3} \times \mathbb{P}^{2} \times \mathbb{P}^{2}}(1,1,1)$ in $Q_{3} \times \mathbb{P}^{2} \times \mathbb{P}^{2}$ has the trivial canonical bundle; therefore, the intersection of three hyperplane classes $\mathcal{O}_{Q_{3} \times \mathbb{P}^{2} \times \mathbb{P}^{2}}(1,1,1)$ gives a Calabi-Yau 4-fold, $Y_{2,2}$.

\footnotetext{
${ }^{4}$ We use Kodaira's notation to denote fiber types. For the Kodaira-Néron classification of the singular fibers of elliptic surfaces, see [57-59].
} 
By construction, Calabi-Yau 4-fold $Y_{2,2}$ has projection $p_{1}$ onto $Q_{3}$. Fiber of this projection is the intersection of three hyperplane classes $\mathcal{O}_{\mathbb{P}^{2} \times \mathbb{P}^{2}}(1,1)$ in $\mathbb{P}^{2} \times \mathbb{P}^{2}$, which is a genus-one curve; therefore, projection $p_{1}$ is a genus-one fibration. Calabi-Yau 4-fold $Y_{2,2}$ also has projection $q_{1}$ onto $\mathbb{P}^{2} \times \mathbb{P}^{2}$. We will find in section 3.3.1 that the pullbacks of hyperplane classes $\mathcal{O}_{\mathbb{P}^{2} \times \mathbb{P}^{2}}(1,0)$ and $\mathcal{O}_{\mathbb{P}^{2} \times \mathbb{P}^{2}}(0,1)$ under projection $q_{1}$ are 3 -sections. Thus, a discrete $\mathbb{Z}_{3}$ symmetry arises in F-theory compactification on Calabi-Yau 4-fold $Y_{2,2}$.

Next, we consider the Segre embedding of $V_{6}=\mathbb{P}^{1} \times \mathbb{P}^{1} \times \mathbb{P}^{1}$ into $\mathbb{P}^{7}$. We consider the direct product of two del Pezzo 3-folds, $V_{6}$ and $V_{E}(E=2, \cdots, 8): V_{6} \times V_{E}$. We take the intersection of two hyperplane classes $\mathcal{O}_{V_{6} \times V_{E}}(1,1)$ in the product $V_{6} \times V_{E}$; the canonical bundle of this intersection has the trivial bundle, thus this construction gives a Calabi-Yau 4-fold, $Y_{1,1,1}$.

By construction, Calabi-Yau 4-fold $Y_{1,1,1}$ has natural projection $p_{2}$ onto $V_{E}$. Fiber of projection $p_{2}$ is the intersection of two hyperplane classes $\mathcal{O}_{V_{6}}(1)$ in $V_{6}$, which is a genus-one curve. Therefore, projection $p_{2}$ gives a genus-one fibration. Calabi-Yau 4 -fold $Y_{1,1,1}$ also has projection $q_{2}$ onto $V_{6}=\mathbb{P}^{1} \times \mathbb{P}^{1} \times \mathbb{P}^{1}$. We will see in section 3.3.2 that the pullbacks of hyperplane classes $\mathcal{O}_{\mathbb{P}^{1} \times \mathbb{P}^{1} \times \mathbb{P}^{1}}(1,0,0), \mathcal{O}_{\mathbb{P}^{1} \times \mathbb{P}^{1} \times \mathbb{P}^{1}}(0,1,0)$ and $\mathcal{O}_{\mathbb{P}^{1} \times \mathbb{P}^{1} \times \mathbb{P}^{1}}(0,0,1)$ under $q_{2}$ are bisections. Therefore, a discrete $\mathbb{Z}_{2}$ symmetry arises in F-theory compactification on $Y_{1,1,1}$.

A similar proof as that given in section 2.1 shows that generic members of the constructed genus-one fibered Calabi-Yau 4-folds $Y_{2,2}$ and $Y_{1,1,1}$ do not have a global section.

\subsection{Hypersurfaces in $\mathbb{P}^{2}$ times Fano 3-folds}

We construct Calabi-Yau 4-folds by considering hypersurfaces in $\mathbb{P}^{2}$ times Fano 3-folds. For Fano 3-folds, we use the following spaces: del Pezzo 3-folds $V_{E}(E=2, \cdots, 8)$, and $\mathbb{P}^{1} \times \mathbb{P}^{2}$.

Hypersurface class $\mathcal{O}_{\mathbb{P}^{2} \times V_{E}}(3,2)$ in the product $\mathbb{P}^{2} \times V_{E}$ is a Calabi-Yau 4-fold, $Y_{4}$. Calabi-Yau 4-fold $Y_{4}$ has natural projection $p$ onto $V_{E}$, and natural projection $q$ onto $\mathbb{P}^{2}$. Fiber of projection $p$ is a cubic hypersurface in $\mathbb{P}^{2}$, which is a genus-one curve. Therefore, projection $p$ is a genus-one fibration. The particular case $E=6$ is a $(3,2,2,2)$ hypersurface in $\mathbb{P}^{2} \times \mathbb{P}^{1} \times \mathbb{P}^{1} \times \mathbb{P}^{1}[36]$.

Similarly, $(3,2,3)$ hypersurfaces in $\mathbb{P}^{2} \times \mathbb{P}^{1} \times \mathbb{P}^{2}$ are genus-one fibered Calabi-Yau 4-folds. Base 3 -fold is $\mathbb{P}^{1} \times \mathbb{P}^{2}$.

We will find in section 3.4 that the pullback of hyperplane class $\mathcal{O}_{\mathbb{P}^{2}}(1)$ under projection $q$ gives a 3 -section. Therefore, a discrete $\mathbb{Z}_{3}$ symmetry arises in F-theory compactifications on these hypersurface constructions of Calabi-Yau 4-folds.

A similar proof as that given in section 2.1 shows that a generic member of the constructed genus-one fibered Calabi-Yau 4-folds $Y_{4}$ does not have a global section.

\subsection{Complete intersections in $\mathbb{P}^{3}$ times Fano 3-folds}

We build Calabi-Yau 4-folds as complete intersections in $\mathbb{P}^{3}$ times Fano 3-folds. Same as in section 2.4, we use del Pezzo 3-folds $V_{E}, E=2, \cdots, 8$, and $\mathbb{P}^{1} \times \mathbb{P}^{2}$, for Fano 3-folds.

Complete intersections of two $\mathcal{O}_{\mathbb{P}^{3} \times V_{E}}(2,1)$ classes in the product $\mathbb{P}^{3} \times V_{E}$ are CalabiYau 4-folds. Fiber of natural projection $p$ onto $V_{E}$ is a complete intersection of two quadrics in $\mathbb{P}^{3}$, which is a genus-one curve. Therefore, projection $p$ gives a genus-one fibration. The particular case $E=6$ is a $(2,1,1,1)$ and $(2,1,1,1)$ complete intersection in $\mathbb{P}^{3} \times \mathbb{P}^{1} \times \mathbb{P}^{1} \times \mathbb{P}^{1}$. 
A similar argument as that stated above shows that $(2,1,2)$ and $(2,1,1)$ complete intersections in $\mathbb{P}^{3} \times \mathbb{P}^{1} \times \mathbb{P}^{2}$ are genus-one fibered Calabi-Yau 4-folds.

Complete intersection in $\mathbb{P}^{3}$ times Fano 3 -folds has natural projection $q$ onto $\mathbb{P}^{3}$. We will find in section 3.5 that the pullback of hyperplane class $\mathcal{O}_{\mathbb{P}^{3}}(1)$ in $\mathbb{P}^{3}$ under $q$ gives a 4 -section. Therefore, we find that a discrete $\mathbb{Z}_{4}$ gauge symmetry arises in F-theory compactifications on genetic members of complete intersections described above.

A similar proof as that given in section 2.1 shows that a generic member of the constructed genus-one fibered Calabi-Yau complete intersections does not have a global section.

We remark that, for special members of $(2,1,1,1)$ and $(2,1,1,1)$ complete intersections in $\mathbb{P}^{3} \times \mathbb{P}^{1} \times \mathbb{P}^{1} \times \mathbb{P}^{1}$, and special members of $(2,1,2)$ and $(2,1,1)$ complete intersections in $\mathbb{P}^{3} \times \mathbb{P}^{1} \times \mathbb{P}^{2}$, given by:

$$
\begin{aligned}
& x_{1}^{2}+x_{3}^{2}+2 \operatorname{tf} x_{2} x_{4}=0 \\
& x_{2}^{2}+x_{4}^{2}+2 \operatorname{tg} x_{1} x_{3}=0 .
\end{aligned}
$$

the discrete symmetry becomes $\mathbb{Z}_{2}$. ([ $\left.x_{1}: x_{2}: x_{3}: x_{4}\right]$ represents homogeneous coordinates on $\mathbb{P}^{3}$. $t$ is the inhomogeneous coordinate on the first $\mathbb{P}^{1}$ in $\mathbb{P}^{3} \times \mathbb{P}^{1} \times \mathbb{P}^{1} \times \mathbb{P}^{1}$, and is the inhomogeneous coordinate on $\mathbb{P}^{1}$ in $\mathbb{P}^{3} \times \mathbb{P}^{1} \times \mathbb{P}^{2}$. For $(2,1,1,1)$ and $(2,1,1,1)$ complete intersections in $\mathbb{P}^{3} \times \mathbb{P}^{1} \times \mathbb{P}^{1} \times \mathbb{P}^{1}, f, g$ are bidegree $(1,1)$ polynomials on $\mathbb{P}^{1} \times \mathbb{P}^{1}$, where this $\mathbb{P}^{1} \times \mathbb{P}^{1}$ is the product of the last two $\mathbb{P}^{1}$ 's in $\mathbb{P}^{3} \times \mathbb{P}^{1} \times \mathbb{P}^{1} \times \mathbb{P}^{1}$. For $(2,1,2)$ and $(2,1,1)$ complete intersections in $\mathbb{P}^{3} \times \mathbb{P}^{1} \times \mathbb{P}^{2}, f, g$ are polynomials of degree 2 and degree 1 on $\mathbb{P}^{2}$, respectively. Simultaneous vanishing of the two equations in (2.12) gives a complete intersection.) $(2,1,1,1)$ and $(2,1,1,1)$ complete intersections, and $(2,1,2)$ and $(2,1,1)$ complete intersections have K3 fibrations, with base surface being $\mathbb{P}^{1} \times \mathbb{P}^{1}$, and $\mathbb{P}^{2}$, respectively. K3 fibers of the complete intersections (2.12) are isomorphic to the Fermat quartic surface, $\left\{x^{4}+y^{4}+z^{4}+w^{4}=0\right\} \subset \mathbb{P}^{3}[35]$. As we will see in section 3.5, for special complete intersections (2.12), 4-sections split into pairs of bisections. As a result, the discrete symmetry that forms in F-theory compactifications becomes $\mathbb{Z}_{2}$.

We prove that the special $(2,1,1,1)$ and $(2,1,1,1)$ complete intersections in $\mathbb{P}^{3} \times \mathbb{P}^{1} \times$ $\mathbb{P}^{1} \times \mathbb{P}^{1}$, and special $(2,1,2)$ and $(2,1,1)$ complete intersections in $\mathbb{P}^{3} \times \mathbb{P}^{1} \times \mathbb{P}^{2}$, both given by $(2.12)$, do not have a rational section. The proof goes as follows: the natural projection of $(2,1,1,1)$ and $(2,1,1,1)$ complete intersection $(2.12)$ onto $\mathbb{P}^{1} \times \mathbb{P}^{1}$, where these $\mathbb{P}^{1}$ 's are the last two $\mathbb{P}^{1}$ 's in the product $\mathbb{P}^{3} \times \mathbb{P}^{1} \times \mathbb{P}^{1} \times \mathbb{P}^{1}$, and the natural projection of $(2,1,2)$ and $(2,1,1)$ complete intersection $(2.12)$ onto $\mathbb{P}^{2}$, have K3 fibers, described by the simultaneous vanishing of the following two equations in $\mathbb{P}^{3} \times \mathbb{P}^{1}$ :

$$
\begin{aligned}
& x_{1}^{2}+x_{3}^{2}+2 t x_{2} x_{4}=0 \\
& x_{2}^{2}+x_{4}^{2}+2 t x_{1} x_{3}=0 .
\end{aligned}
$$

K3 fiber (2.13) is isomorphic to the Fermat quartic surface. K3 fiber $(2.13)$ is a $(2,1)$ and $(2,1)$ complete intersection in $\mathbb{P}^{3} \times \mathbb{P}^{1}$; therefore, it has natural projection onto $\mathbb{P}^{1}$, which gives a genus-one fibration of K3 surface (2.13). This genus-one fibration does not have a global section [35]. Therefore, K3 fibers (2.13) are genus-one fibered, but they lack a global section. If $(2,1,1,1)$ and $(2,1,1,1)$ complete intersection $(2.12)$, and $(2,1,2)$ and $(2,1,1)$ 
complete intersection (2.12), have rational sections, these rational sections restrict to K3 fibers, giving global sections to K3 fibers (2.13). Therefore, the fact that K3 fibers (2.13) lack a global section implies that $(2,1,1,1)$ and $(2,1,1,1)$ complete intersection Calabi-Yau 4 -folds $(2.12)$ and $(2,1,2)$ and $(2,1,1)$ complete intersection Calabi-Yau 4-folds (2.12) do not admit a rational section.

\subsection{Double covers of $\mathbb{P}^{1}$ times Fano 3 -folds}

The following double covers of the products $\mathbb{P}^{1}$ times Fano 3 -folds are Calabi-Yau 4-folds:

- double covers of $\mathbb{P}^{1} \times V_{E}$ ramified over a $\mathcal{O}_{\mathbb{P}^{1} \times V_{E}}(4,4) 3$-fold

- double covers of $\mathbb{P}^{1} \times \mathbb{P}^{1} \times \mathbb{P}^{2}$ ramified over a $(4,4,6) 3$-fold

Each of these double covers has projection $p$ onto the base 3 -folds $B_{3}$, where the base 3 -fold $B_{3}$ is the del Pezzo 3-fold of degree $E, V_{E}$ for the first double cover construction, and the base 3 -fold $B_{3}$ is $\mathbb{P}^{1} \times \mathbb{P}^{2}$ for the second double cover construction, respectively. Fiber of projection $p$ is a double cover of $\mathbb{P}^{1}$ ramified over 4 point, which is a genus-one curve. Thus, projection $p$ gives a genus-one fibration.

These double covers have natural projection $q$ onto $\mathbb{P}^{1}$. We will see in section 3.6 that the pullback of point class $\mathcal{O}_{\mathbb{P}^{1}}(1)$ in $\mathbb{P}^{1}$ under $q$ gives a bisection. Therefore, a discrete $\mathbb{Z}_{2}$ symmetry arises in F-theory compactifications on these double covers.

\section{Discrete gauge symmetry}

In this section, we represent multisections of the Calabi-Yau genus-one fibrations as the pullbacks of hyperplane classes from the spaces in which genus-one fibers embed. By computing the intersection numbers of these pullbacks with fiber classes $F$, we determine the degrees of the multisections. Using this method, we deduce the discrete gauge symmetries that arise in F-theory compactifications on the six constructions of genus-one fibered Calabi-Yau 4-folds without a section that we introduced in section 2.

\subsection{Discrete $\mathbb{Z}_{2}, \mathbb{Z}_{3}, \mathbb{Z}_{4}$ and $\mathbb{Z}_{5}$ symmetries on intersections of two hyperplane classes in product of del Pezzo 3-folds}

In section 2.1, we constructed genus-one fibered Calabi-Yau 4-fold $Y_{4}$ as the intersection of two hyperplane sections $\mathcal{O}_{V_{D} \times V_{E}}(1,1)$ in the product of two del Pezzo 3 -folds, $V_{D} \times V_{E}$, $D=2,3,4,5, E=2, \cdots, 8$.

By construction, Calabi-Yau 4-fold $Y_{4}$ has natural projection $p$ onto $V_{E}$, and natural projection $q$ onto $V_{D}$. We use $V_{E}$ as the base 3 -fold. Projection $p$ gives a genus-one fibration; genus-one fiber is the intersection of two hyperplane classes $\mathcal{O}_{V_{D}}(1)$ in $V_{D}$. The pullback of a point class in the base 3 -fold $V_{E}, p^{*}\{\mathrm{pt}\}$, has self-intersection 0 ; therefore it represents the fiber class $F$. The pullback of a hyperplane class $\mathcal{O}_{V_{D}}(1)$ in $V_{D}, q^{*} \mathcal{O}_{V_{D}}(1)$, has an intersection number $D$ with fiber class $F=p^{*}\{\mathrm{pt}\}$, where $D$ is the degree of the del Pezzo 3-fold $V_{D}$. Thus, pullback

$$
q^{*} \mathcal{O}_{V_{D}}(1)
$$

represents a $D$-section, $D=2,3,4,5$. 
Therefore, we conclude that a discrete $\mathbb{Z}_{5}$ symmetry arises in F-theory compactifications on intersections of two hyperplane sections in $V_{5} \times V_{E}$. Discrete $\mathbb{Z}_{4}, \mathbb{Z}_{3}$ and $\mathbb{Z}_{2}$ symmetries arise in F-theory compactifications on intersections of two hyperplane sections in $V_{4} \times V_{E}, V_{3} \times V_{E}$ and $V_{2} \times V_{E}$, respectively.

Additionally, as stated in section 2.1, when $D=8$ multisection $q^{*} \mathcal{O}_{V_{8}}(1)$ represents a 4section. Thus, a discrete $\mathbb{Z}_{4}$ symmetry forms in F-theory compactifications on intersections of two hyperplane sections in $V_{8} \times V_{E}$.

As stated in section 2.1, the base 3 -fold $V_{E}$ may be replaced by $\mathbb{P}^{1} \times \mathbb{P}^{2}$. The results obtained above remain unchanged when the base 3 -fold is replaced by $\mathbb{P}^{1} \times \mathbb{P}^{2}$.

\subsection{Discrete $\mathbb{Z}_{3}$ symmetry on cyclic 3 -fold covers of $\mathbb{P}^{1}$ times del Pezzo 3-folds}

Cyclic 3 -fold covers of $\mathbb{P}^{1} \times V_{E}$ ramified over a $\mathcal{O}_{\mathbb{P}^{1} \times V_{E}}(3,3) 3$-fold are Calabi-Yau 4-folds. $V_{E}$ denotes the del Pezzo 3-fold of degree $E$, with $E=2, \cdots, 8$. Such cyclic 3-fold covers have projection $p$ onto $V_{E}$, and projection $q$ onto $\mathbb{P}^{1}$. Projection $p$ gives a genus-one fibration; genus-one fiber is a cyclic 3 -fold cover of a rational curve $\mathbb{P}^{1}$ ramified over 3 points.

The pullback of a point class in the base 3 -fold $V_{E}, p^{*}\{\mathrm{pt}\}$, has self-intersection 0 ; therefore it gives the fiber class $F$. The pullback of a point class $\mathcal{O}_{\mathbb{P}^{1}}(1)$ in $\mathbb{P}^{1}, q^{*} \mathcal{O}_{\mathbb{P}^{1}}(1)$, has an intersection number 3 with fiber class $F=p^{*}\{\mathrm{pt}\}$. Therefore, we conclude that the multisection

$$
q^{*} \mathcal{O}_{\mathbb{P}^{1}}(1)
$$

is a 3 -section. Thus, a discrete $\mathbb{Z}_{3}$ gauge symmetry forms in F-theory compactifications on cyclic 3 -fold covers of $\mathbb{P}^{1} \times V_{E}$ ramified over a $\mathcal{O}_{\mathbb{P}^{1} \times V_{E}}(3,3) 3$-fold.

\subsection{Discrete $\mathbb{Z}_{3}$ and $\mathbb{Z}_{2}$ symmetries on constructions involving Segre embed- dings of $\mathbb{P}^{2} \times \mathbb{P}^{2}$ and $\mathbb{P}^{1} \times \mathbb{P}^{1} \times \mathbb{P}^{1}$}

\subsection{1 $\mathbb{Z}_{3}$ symmetry in constructions involving Segre embeddings of $\mathbb{P}^{2} \times \mathbb{P}^{2}$ into $\mathbb{P}^{8}$}

We consider the Segre embedding of $\mathbb{P}^{2} \times \mathbb{P}^{2}$ into $\mathbb{P}^{8}: \mathbb{P}^{2} \times \mathbb{P}^{2} \hookrightarrow \mathbb{P}^{8} . Q_{3}$ is a quadric 3 -fold in $\mathbb{P}^{4}$. We saw in section 2.3 that the intersection of three hyperplane sections $\mathcal{O}_{Q_{3} \times \mathbb{P}^{2} \times \mathbb{P}^{2}}(1,1,1)$ in the product $Q_{3} \times \mathbb{P}^{2} \times \mathbb{P}^{2}$ is a Calabi-Yau 4-fold, $Y_{2,2}$. $Y_{2,2}$ has projection $p_{1}$ onto $Q_{3}$, and projection $q_{1}$ onto $\mathbb{P}^{2} \times \mathbb{P}^{2}$. Projection $p_{1}$ gives a genus-one fibration; genus-one fiber is the intersection of three hyperplane classes $\mathcal{O}_{\mathbb{P}^{2} \times \mathbb{P}^{2}}(1,1)$ in $\mathbb{P}^{2} \times \mathbb{P}^{2}$.

The pullback of a point class in the base 3 -fold $Q_{3}, p_{1}^{*}\{\mathrm{pt}\}$, represents the fiber class $F$. The pullback of hyperplane class $\mathcal{O}_{\mathbb{P}^{2} \times \mathbb{P}^{2}}(1,0)$ in $\mathbb{P}^{2} \times \mathbb{P}^{2}, q_{1}^{*} \mathcal{O}_{\mathbb{P}^{2} \times \mathbb{P}^{2}}(1,0)$, has an intersection number 3 with fiber class $F$. Similarly, pullback $q_{1}^{*} \mathcal{O}_{\mathbb{P}^{2} \times \mathbb{P}^{2}}(0,1)$ has an intersection number 3 with fiber class $F$. Therefore, we find that pullbacks

$$
q_{1}^{*} \mathcal{O}_{\mathbb{P}^{2} \times \mathbb{P}^{2}}(1,0), \quad q_{1}^{*} \mathcal{O}_{\mathbb{P}^{2} \times \mathbb{P}^{2}}(0,1)
$$

represent 3-sections. A discrete $\mathbb{Z}_{3}$ gauge group arises in F-theory compactification on $Y_{2,2}$. 


\subsection{2 $\mathbb{Z}_{2}$ symmetry in constructions involving Segre embeddings of $\mathbb{P}^{1} \times \mathbb{P}^{1} \times \mathbb{P}^{1}$ into $\mathbb{P}^{7}$}

We consider the Segre embedding of $V_{6}=\mathbb{P}^{1} \times \mathbb{P}^{1} \times \mathbb{P}^{1}$ into $\mathbb{P}^{7}: \mathbb{P}^{1} \times \mathbb{P}^{1} \times \mathbb{P}^{1} \hookrightarrow \mathbb{P}^{7}$. Intersection of two hyperplane sections $\mathcal{O}_{V_{6} \times V_{E}}(1,1)$ in the product of two del Pezzo 3folds, $V_{6} \times V_{E}, E=2, \cdots, 8$, is a Calabi-Yau 4-fold, $Y_{1,1,1}$. $Y_{1,1,1}$ has projection $p_{2}$ onto $V_{E}$, and projection $q_{2}$ onto $V_{6}$. Projection $p_{2}$ is a genus-one fibration, and genus-one fiber is the intersection of two hyperplanes $\mathcal{O}_{V_{6}}(1)$ in $V_{6}=\mathbb{P}^{1} \times \mathbb{P}^{1} \times \mathbb{P}^{1}$.

The pullback of a point class in the base 3 -fold $V_{E}, p_{2}^{*}\{\mathrm{pt}\}$, gives the fiber class $F$. The pullback of hyperplane class $\mathcal{O}_{\mathbb{P}^{1} \times \mathbb{P}^{1} \times \mathbb{P}^{1}}(1,0,0)$ in $V_{6}=\mathbb{P}^{1} \times \mathbb{P}^{1} \times \mathbb{P}^{1}, q_{2}^{*} \mathcal{O}_{\mathbb{P}^{1} \times \mathbb{P}^{1} \times \mathbb{P}^{1}}(1,0,0)$, has an intersection number 2 with fiber class $F$. Similarly, Pullbacks $q_{2}^{*} \mathcal{O}_{\mathbb{P}^{1} \times \mathbb{P}^{1} \times \mathbb{P}^{1}}(0,1,0)$ and $q_{2}^{*} \mathcal{O}_{\mathbb{P}^{1} \times \mathbb{P}^{1} \times \mathbb{P}^{1}}(0,0,1)$ have intersection number 2 with fiber class $F$. Therefore, pullbacks

$$
q_{2}^{*} \mathcal{O}_{\mathbb{P}^{1} \times \mathbb{P}^{1} \times \mathbb{P}^{1}}(1,0,0), \quad q_{2}^{*} \mathcal{O}_{\mathbb{P}^{1} \times \mathbb{P}^{1} \times \mathbb{P}^{1}}(0,1,0), \quad q_{2}^{*} \mathcal{O}_{\mathbb{P}^{1} \times \mathbb{P}^{1} \times \mathbb{P}^{1}}(0,0,1)
$$

are bisections. Thus, a discrete $\mathbb{Z}_{2}$ gauge group forms in F-theory compactification on $Y_{1,1,1}$.

\subsection{Discrete $\mathbb{Z}_{3}$ symmetry on hypersurfaces in $\mathbb{P}^{2}$ times Fano 3-folds}

In section 2.4, we considered the following two constructions of Calabi-Yau 4-fold as hypersurfaces in $\mathbb{P}^{2}$ times Fano 3-folds:

- Hypersurfaces of class $\mathcal{O}_{\mathbb{P}^{2} \times V_{E}}(3,2)$ in $\mathbb{P}^{2} \times V_{E}$

- $(3,2,3)$ Hypersurfaces in $\mathbb{P}^{2} \times \mathbb{P}^{1} \times \mathbb{P}^{2}$

These Calabi-Yau hypersurfaces have projection $p$ onto $B_{3}$, and projection $q$ onto $\mathbb{P}^{2}$. For the first hypersurface, $B_{3}$ is the del Pezzo 3 -fold $V_{E}$, and for the second hypersurface, $B_{3}$ is $\mathbb{P}^{1} \times \mathbb{P}^{2}$. Projection $p$ gives a genus-one fibration; a genus-one fiber is a cubic hypersurface in $\mathbb{P}^{2}$. The argument that follows to determine the degree of a multisection does not depend on the structure of $B_{3}$.

The pullback of a point class in the base 3 -fold $B_{3}, p^{*}\{\mathrm{pt}\}$, has self-intersection 0 ; therefore, it gives the fiber class $F$. The pullback of a line class $\mathcal{O}_{\mathbb{P}^{2}}(1)$ in $\mathbb{P}^{2}, q^{*} \mathcal{O}_{\mathbb{P}^{2}}(1)$, has an intersection number 3 with fiber class $F$. Therefore, pullback

$$
q^{*} \mathcal{O}_{\mathbb{P}^{2}}(1)
$$

represents a 3 -section. A discrete $\mathbb{Z}_{3}$ symmetry arises in F-theory compactifications on these Calabi-Yau hypersurfaces.

\subsection{Discrete $\mathbb{Z}_{4}, \mathbb{Z}_{2}$ symmetries on complete intersections in $\mathbb{P}^{3}$ times Fano 3-folds}

In section 2.5, we considered the following complete intersections to construct Calabi-Yau 4-folds:

- Complete Intersections of two $\mathcal{O}_{\mathbb{P}^{3} \times V_{E}}(2,1)$ hypersurfaces in $\mathbb{P}^{3} \times V_{E}$

- Complete Intersections of $(2,1,2)$ and $(2,1,1)$ hypersurfaces in $\mathbb{P}^{3} \times \mathbb{P}^{1} \times \mathbb{P}^{2}$. 
By construction, these complete intersection Calabi-Yau 4-folds have projection $p$ onto $B^{3}$, and projection $q$ onto $\mathbb{P}^{3} . B_{3}$ is the del Pezzo 3 -fold $V_{E}, E=2, \cdots, 8$, for the first complete intersection, and $B_{3}$ is $\mathbb{P}^{1} \times \mathbb{P}^{2}$ for the second complete intersection. Projection $p$ gives a genus-one fibration; a genus-one fiber is a complete intersection of two quadrics in $\mathbb{P}^{3}$. The argument that follows to determine the degree of a multisection does not depend on the structure of $B_{3}$.

The pullback of a point class in the base 3 -fold $B_{3}, p^{*}\{\mathrm{pt}\}$, represents the fiber class $F$. The pullback of a surface class $\mathcal{O}_{\mathbb{P}^{3}}(1)$ in $\mathbb{P}^{3}, q^{*} \mathcal{O}_{\mathbb{P}^{3}}(1)$, has an intersection number 4 with fiber class $F$. Therefore, pullback

$$
q^{*} \mathcal{O}_{\mathbb{P}} 3(1)
$$

represents a 4 -section. A discrete $\mathbb{Z}_{4}$ gauge symmetry arises in F-theory compactifications on generic members of these complete intersections.

We remark that for special members of complete intersections, 4-sections split into pairs of bisections. We consider the special $(2,1,1,1)$ and $(2,1,1,1)$ complete intersections in $\mathbb{P}^{3} \times$ $\mathbb{P}^{1} \times \mathbb{P}^{1} \times \mathbb{P}^{1}$, and special $(2,1,2)$ and $(2,1,1)$ complete intersections in $\mathbb{P}^{3} \times \mathbb{P}^{1} \times \mathbb{P}^{2}$, given by

$$
\begin{aligned}
& x_{1}^{2}+x_{3}^{2}+2 t f x_{2} x_{4}=0 \\
& x_{2}^{2}+x_{4}^{2}+2 \operatorname{tg} x_{1} x_{3}=0 .
\end{aligned}
$$

We observe that for the special complete intersections (3.7), 4-sections split into pairs of bisections. To observe this explicitly, we consider the following locus:

$$
x_{1}=0 \text {. }
$$

Along the locus (3.8), the second equation in (3.7) becomes

$$
x_{2}^{2}+x_{4}^{2}=0
$$

which is independent of $t$ and $f, g$, i.e., independent of the coordinates on the base 3 -fold $B_{3}$. Equation (3.9) splits into linear factors as follows:

$$
x_{2}^{2}+x_{4}^{2}=\left(x_{2}+i x_{4}\right)\left(x_{2}-i x_{4}\right)=0,
$$

from which we find that $\left\{x_{1}=0, x_{2}+i x_{4}=0\right\}$ and $\left\{x_{1}=0, x_{2}-i x_{4}=0\right\}$ give bisections of a complete intersection (3.7). This implies that a 4-section splits into a pair of two bisections, $\left\{x_{1}=0, x_{2}+i x_{4}=0\right\}$ and $\left\{x_{1}=0, x_{2}-i x_{4}=0\right\}$, for a complete intersection (3.7). By applying a similar argument to three other loci, $x_{2}=0, x_{3}=0$, and $x_{4}=0$, we observe that a complete intersection (3.7) has eight bisections. (We do not know if a complete intersection (3.7) has more bisections.)

In summary, we have shown that the special complete intersections (3.7) have bisections to the fibration, and from this result we deduce that a discrete $\mathbb{Z}_{2}$ gauge group arises in F-theory compactifications on the special complete intersections (3.7). 


\subsection{Discrete $\mathbb{Z}_{2}$ symmetry on double covers of $\mathbb{P}^{1}$ times Fano 3-folds}

In section 2.6, we considered the following constructions of Calabi-Yau 4-folds as double covers of the products of $\mathbb{P}^{1}$ times Fano 3-folds:

- double covers of $\mathbb{P}^{1} \times V_{E}$ ramified over a $\mathcal{O}_{\mathbb{P}^{1} \times V_{E}}(4,4) 3$-fold

- double covers of $\mathbb{P}^{1} \times \mathbb{P}^{1} \times \mathbb{P}^{2}$ ramified over a $(4,4,6) 3$-fold.

By construction, these Calabi-Yau double covers have projection $p$ onto $B_{3}$, and projection $q$ onto $\mathbb{P}^{1}$. The base 3 -fold $B_{3}$ is the del Pezzo 3-fold of degree $E, V_{E}$ for the first double cover construction, and $B_{3}$ is $\mathbb{P}^{1} \times \mathbb{P}^{2}$ for the second construction. The projection $p$ gives a genus-one fibration; genus-one fiber is a double cover of $\mathbb{P}^{1}$ ramified over 4 points. The argument that follows to determine the degree of a multisection does not depend on the structure of $B_{3}$.

The pullback of a point class in the base 3 -fold $B_{3}, p^{*}\{\mathrm{pt}\}$, has self-intersection 0 ; therefore, it represents the fiber class $F$. The pullback of a point class in $\mathbb{P}^{1}, q^{*} \mathcal{O}_{\mathbb{P}^{1}}(1)$, has an intersection number 2 with fiber class $F$. Therefore, pullback

$$
q^{*} \mathcal{O}_{\mathbb{P}^{1}}(1)
$$

is a bisection. A discrete $\mathbb{Z}_{2}$ symmetry arises in F-theory compactifications on these CalabiYau double covers.

\section{Conclusion}

In this note, we investigated the discrete gauge symmetries that arise in F-theory compactifications on examples of genus-one fibered Calabi-Yau 4-folds without a section. We constructed genus-one fibered Calabi-Yau 4-folds that do not have a global section, by considering constructions using Fano manifolds, double covers, cyclic 3-fold covers and Segre embeddings of products of projective spaces. For these constructions, we obtained multisections as the pullbacks of hyperplanes classes in spaces in which fibers embed. We determined the degrees of these multisections by computing the intersection numbers with the fiber classes $F$, and we deduced the discrete gauge symmetries that form in F-theory compactifications on the six constructions of Calabi-Yau genus-one fibrations.

In particular, the Calabi-Yau constructions that use the del Pezzo 3-folds (section 2.1), cyclic 3-fold covers (section 2.2), and the Segre embeddings of products of projective spaces (section 2.3) provide novel examples of F-theory compactifications in which discrete gauge groups arise. Discrete $\mathbb{Z}_{5}, \mathbb{Z}_{4}, \mathbb{Z}_{3}$ and $\mathbb{Z}_{2}$ symmetries arise in the constructions that use the product of del Pezzo 3-folds, $V_{D} \times V_{E}$, depending on the degree of the del Pezzo 3-fold $V_{D}$. A discrete $\mathbb{Z}_{3}$ symmetry arises in F-theory compactifications on cyclic 3-fold covers of $\mathbb{P}^{1} \times V_{E}$ ramified over a $\mathcal{O}_{\mathbb{P}^{1} \times V_{E}}(3,3) 3$-fold. A discrete $\mathbb{Z}_{3}$ symmetry arises in F-theory compactifications on the Calabi-Yau constructions that use the Segre embedding of $\mathbb{P}^{2} \times \mathbb{P}^{2}$ into $\mathbb{P}^{8}$. A discrete $\mathbb{Z}_{2}$ symmetry arises in F-theory compactifications on the Calabi-Yau constructions that use the Segre embedding of $\mathbb{P}^{1} \times \mathbb{P}^{1} \times \mathbb{P}^{1}$ into $\mathbb{P}^{7}$. 
For special complete intersection Calabi-Yau 4-folds in $\mathbb{P}^{3} \times \mathbb{P}^{1} \times \mathbb{P}^{1} \times \mathbb{P}^{1}$, and special complete intersection Calabi-Yau 4-folds in $\mathbb{P}^{3} \times \mathbb{P}^{1} \times \mathbb{P}^{2}$, whose K3 fibers are isomorphic to the Fermat quartic, we observed that 4-sections split into pairs of bisections. Consequently, the discrete symmetry that arises in F-theory compactifications becomes $\mathbb{Z}_{2}$ for these special cases.

The method, introduced in section 3 to determine the discrete gauge symmetries that arise in F-theory compactifications, is considerably general, and is applied to all six constructions of Calabi-Yau 4-folds. The same method applies to similar constructions of Calabi-Yau genus-one fibrations.

\section{Acknowledgments}

We would like to thank Shigeru Mukai and Fumihiro Takayama for discussions. We are also grateful to the referee for improving this manuscript.

\section{A Gauge theories and matter spectra on $(2,1,1,1)$ and $(2,1,1,1)$ complete intersections in $\mathbb{P}^{3} \times \mathbb{P}^{1} \times \mathbb{P}^{1} \times \mathbb{P}^{1}$}

We determine the forms of the discriminant components, and the non-Abelian gauge groups arising on the 7-branes in F-theory compactifications on the special $(2,1,1,1)$ and $(2,1,1,1)$ complete intersections (2.12) in $\mathbb{P}^{3} \times \mathbb{P}^{1} \times \mathbb{P}^{1} \times \mathbb{P}^{1}$. We derive the equations of the Jacobian fibrations and determine the Mordell-Weil group of the Jacobians. We find that the Mordell-Weil group has rank 0; therefore, F-theory models on the special $(2,1,1,1)$ and $(2,1,1,1)$ complete intersection Calabi-Yau 4-folds (2.12) do not have a U(1) gauge symmetry. The relationship between the gauge symmetry and the torsion part of the Mordell-Weil group is discussed in $[60,61]$. By computing the Euler characteristic of the complete intersections (2.12), we derive a condition imposed on a 4 -form flux ${ }^{5}$ to cancel the tadpole. As discussed in [36], to determine whether a consistent flux [32, 71-74] exists, we need to compute the self-intersections of intrinsic 2-cycles, which is technically considerably difficult. For this reason, in this study, we do not consider whether a consistent flux exists. We compute the potential matter spectra and potential Yukawa couplings. As it is undetermined whether a consistent flux choice exists, the contents of the spectra are potential matter and they could arise. Similar organization can be found in [36].

\section{A.1 Forms of the discriminant components, non-Abelian gauge groups on 7- branes and Jacobian fibration}

In section 2.5 and 3.5 , we discussed the special $(2,1,1,1)$ and $(2,1,1,1)$ complete intersections in $\mathbb{P}^{3} \times \mathbb{P}^{1} \times \mathbb{P}^{1} \times \mathbb{P}^{1}$, given by

$$
\begin{aligned}
& x_{1}^{2}+x_{3}^{2}+2 \operatorname{tf} x_{2} x_{4}=0 \\
& x_{2}^{2}+x_{4}^{2}+2 \operatorname{tg} x_{1} x_{3}=0 .
\end{aligned}
$$

\footnotetext{
${ }^{5}[62]$ studied 4-form flux. For recent studies of 4-form fluxes in F-theory, see, for example, [6, 63-70].
} 
The K3 fibers of the complete intersection Calabi-Yau 4-folds (A.1) are isomorphic to the Fermat quartic. Using a method similar to that in [35], we derive the equation of the Jacobian fibrations of the complete intersections (A.1).

We introduce a parameter $\lambda$, and subtract $\lambda$ times the second equation from the first equation in (A.1), as follows:

$$
x_{1}^{2}+x_{3}^{2}+2 t f x_{2} x_{4}-\lambda\left(x_{2}^{2}+x_{4}^{2}+2 \operatorname{tg} x_{1} x_{3}\right) .
$$

We arrange the coefficients of equation (A.2) into a symmetric $4 \times 4$ matrix, and compute the determinant of the matrix to obtain the equation of the Jacobian fibrations of the complete intersections (A.1) as follows:

$$
\tau^{2}=-t^{2} g^{2} \lambda^{4}+\left(t^{4} f^{2} g^{2}+1\right) \lambda^{2}-t^{2} f^{2} .
$$

The Jacobian fibrations (A.3) describe double covers of $\mathbb{P}^{1} \times \mathbb{P}^{1} \times \mathbb{P}^{1} \times \mathbb{P}^{1}$, where $\lambda$ and $t$ are the inhomogeneous coordinates on the first and the second $\mathbb{P}^{1}$ 's, respectively, in $\mathbb{P}^{1} \times \mathbb{P}^{1} \times \mathbb{P}^{1} \times \mathbb{P}^{1}$, and $f, g$ are $(1,1)$ polynomials on $\mathbb{P}^{1} \times \mathbb{P}^{1}$. (This $\mathbb{P}^{1} \times \mathbb{P}^{1}$ is the product of the third and the fourth $\mathbb{P}^{1}$ 's in $\mathbb{P}^{1} \times \mathbb{P}^{1} \times \mathbb{P}^{1} \times \mathbb{P}^{1}$.) The Jacobians (A.3) are Calabi-Yau 4-folds.

A complete intersection (A.1) and its Jacobian fibration (A.3) have the same discriminant loci and fiber types. Therefore, we can determine the forms of the discriminant components and the non-Abelian gauge groups in F-theory compactifications on the complete intersections (A.1) by studying the Jacobian fibrations (A.3).

The discriminant of the Jacobian (A.3) is given by

$$
\begin{aligned}
\Delta & =16 f^{2} g^{2} t^{4}\left(f^{2} g^{2} t^{4}-1\right)^{4} \\
& =16 f^{2} g^{2} t^{4}\left(f g t^{2}-1\right)^{4}\left(f g t^{2}+1\right)^{4}
\end{aligned}
$$

We can deduce the discriminant components from the vanishing of discriminant $\Delta$ (A.4) as follows:

$$
\begin{aligned}
E_{1} & :=\{t=0\} \\
E_{2} & :=\{t=\infty\} \\
D_{1} & :=\{f=0\} \\
D_{2} & :=\{g=0\} \\
D_{3} & :=\{u=\infty\} \\
D_{4} & :=\{v=\infty\} \\
D_{5} & :=\left\{f g t^{2}=1\right\} \\
D_{6} & :=\left\{f g t^{2}=-1\right\} .
\end{aligned}
$$

In (A.5), we have used notations $u$ and $v$ to denote the inhomogeneous coordinates on the second and third $\mathbb{P}^{1}$ 's, respectively, in the base 3 -fold, $\mathbb{P}^{1} \times \mathbb{P}^{1} \times \mathbb{P}^{1}$. With these notations, $f, g$ are bidegree $(1,1)$ polynomials in two variables, $u$ and $v$.

We have

$$
E_{i} \cong \mathbb{P}^{1} \times \mathbb{P}^{1} \quad(i=1,2)
$$




\begin{tabular}{|c|c|}
\hline Component & Topology \\
\hline$E_{i}(i=1,2)$ & $\mathbb{P}^{1} \times \mathbb{P}^{1}$ \\
$D_{i}(i=1,2,3,4)$ & $\mathbb{P}^{1} \times \mathbb{P}^{1}$ \\
\hline Intersections & \\
\hline$D_{1} \cap D_{2}$ & parallel $2 \mathbb{P}^{1} \mathrm{~s}$ \\
$D_{3} \cap D_{4}$ & $\mathbb{P}^{1}$ \\
$E_{i} \cap D_{j}(i=1,2, j=1,2,3,4)$ & $\mathbb{P}^{1}$ \\
\hline
\end{tabular}

Table 2. Discriminant components of a complete intersection (A.1) of two $(2,1,1,1)$ hypersurfaces in $\mathbb{P}^{3} \times \mathbb{P}^{1} \times \mathbb{P}^{1} \times \mathbb{P}^{1}$, and their intersections. Components $D_{5}$ and $D_{6}$ are omitted.

and

$$
D_{i} \cong \mathbb{P}^{1} \times \mathbb{P}^{1} \quad(i=3,4) .
$$

$E_{1}$ and $E_{2}$ are parallel. $D_{3} \cap D_{4}$ is $\mathbb{P}^{1}$. We have

$$
E_{i} \cap D_{j} \cong \mathbb{P}^{1} \quad(i=1,2, \quad j=3,4) .
$$

A bidegree $(1,1)$ curve in $\mathbb{P}^{1} \times \mathbb{P}^{1}$ is a rational curve $\Sigma_{0} \cong \mathbb{P}^{1}$. Therefore,

$$
D_{i} \cong \mathbb{P}^{1} \times \Sigma_{0} \cong \mathbb{P}^{1} \times \mathbb{P}^{1} \quad(i=1,2)
$$

and

$$
E_{i} \cap D_{j} \cong \Sigma_{0} \cong \mathbb{P}^{1} \quad(i, j=1,2) .
$$

Two bidegree $(1,1)$ curves in $\mathbb{P}^{1} \times \mathbb{P}^{1}$ intersect at 2 points; thus, $D_{1} \cap D_{2}$ is a disjoint sum of $2 \mathbb{P}^{1}$ 's. We omit components $D_{5,6}$. We display the forms of the irreducible components, $E_{i}(i=1,2)$ and $D_{i}(i=1,2,3,4)$, of the discriminant locus, and the forms of their intersections in table 2 .

We derive the non-Abelian gauge symmetries on the 7-branes. The Jacobian fibration (A.3) transforms into the extended Weierstrass form given by the equation

$$
y^{2}=\frac{1}{4} x^{3}-\frac{1}{2}\left(f^{2} g^{2} t^{4}+1\right) x^{2}+\frac{1}{4}\left(f^{2} g^{2} t^{4}-1\right)^{2} x
$$

From the extended Weierstrass form, one can determine if a singular fiber is multiplicative (which corresponds to $I_{n}$ fibers) or additive (which corresponds to the other fiber types, i.e. $\left.I I I, I V, I I^{*}, I I I^{*}, I V^{*}, I_{m}^{*}\right)$, by studying the coefficient of $x^{2}{ }^{6}$ For example, when a singular fiber is at $t$, under some appropriate translation in $x$, an extended Weierstrass form transforms into another extended Weierstrass form $y^{2}=x^{3}+a_{2} x^{2}+a_{4} x+a_{6}$ in such a way that $t$ divides $a_{4}$ and $a_{6}$; when $t$ does not divide $a_{2}$, the fiber type at $t$ is multiplicative, and when $t$ divides $a_{2}$, the fiber type at $t$ is additive.

\footnotetext{
${ }^{6}$ See, for example, [75].
} 
The discriminant $\Delta$ (A.4) vanishes under the conditions

$$
f g t^{2}=0, \quad f g t^{2}-1=0, \quad f g t^{2}+1=0,
$$

and these conditions specify the locations of the singular fibers. The coefficient of $x^{2}$ in the extended Weierstrass form (A.11)

$$
-\frac{1}{2}\left(f^{2} g^{2} t^{4}+1\right)
$$

does not vanish under the conditions

$$
f g t^{2}-1=0, \quad f g t^{2}+1=0
$$

in (A.12). Under the translation in $x$ that replaces $x$ with $x+1$, the extended Weierstrass form (A.11) transforms into

$$
y^{2}=\frac{1}{4} x^{3}+\left(\frac{1}{4}-\frac{1}{2} f^{2} g^{2} t^{4}\right) x^{2}+\left(\frac{1}{4} f^{4} g^{4} t^{8}-\frac{3}{2} f^{2} g^{2} t^{4}\right) x+\frac{1}{4} f^{4} g^{4} t^{8}-f^{2} g^{2} t^{4} .
$$

The coefficient of $x^{2}$ in the extended Weierstrass form (A.15)

$$
\frac{1}{4}-\frac{1}{2} f^{2} g^{2} t^{4}
$$

does not vanish under the condition

$$
f g t^{2}=0
$$

in (A.12). Thus, we conclude that singular fibers are multiplicative. Therefore, the fiber types are $I_{n}$ for some $n .^{7}$ The fiber types can be determined from the multiplicities of the zeros of discriminant $\Delta$ (A.4). The fiber type on components $E_{1}$ and $E_{2}$ is $I_{4}$. We consider the translation in $y, \tilde{y}=y+\left(t+\frac{1}{2}\right) x+f g t^{2}$, for extended Weierstrass form (A.15) to see that $I_{4}$ fibers on components $E_{1}$ and $E_{2}$ are of split type. The corresponding gauge groups on the 7-branes wrapped on components $E_{1}$ and $E_{2}$ are $\mathrm{SU}(4)$. The fiber type on components $D_{1}$ and $D_{2}$ is $I_{2}$. Similarly, the fiber type on components $D_{3}$ and $D_{4}$ is $I_{2}$. The corresponding gauge groups on the 7-branes wrapped on $D_{1}, D_{2}, D_{3}$, and $D_{4}$ are $\mathrm{SU}(2)$. The fiber type on components $D_{5}$ and $D_{6}$ is $I_{4}$. We consider the translation in $y, \tilde{y}=y-x$, for extended Weierstrass form (A.11) to find that $I_{4}$ fibers on components $D_{5}$ and $D_{6}$ are of split type. The corresponding gauge groups on the 7 -branes wrapped on $D_{5}$ and $D_{6}$ are $\mathrm{SU}(4)$. We show the results in table 3.

\section{A.2 Mordell-Weil group of the Jacobian fibration}

In A.1, we deduced that the Jacobian fibration of a complete intersection Calabi-Yau 4fold (A.1) is given by the equation

$$
\tau^{2}=-t^{2} g^{2} \lambda^{4}+\left(t^{4} f^{2} g^{2}+1\right) \lambda^{2}-t^{2} f^{2} .
$$

\footnotetext{
${ }^{7}$ One can also see this fact by completing the cube to transform the extended Weierstrass form (A.11) into the Weierstrass form, and by studying the coefficients of the obtained Weierstrass form.
} 


\begin{tabular}{|c|c|c|}
\hline Component & Fiber type & non-Abel. Gauge Group \\
\hline$E_{1,2}$ & $I_{4}$ & $\mathrm{SU}(4)$ \\
\hline$D_{1,2,3,4}$ & $I_{2}$ & $\mathrm{SU}(2)$ \\
\hline$D_{5,6}$ & $I_{4}$ & $\mathrm{SU}(4)$ \\
\hline
\end{tabular}

Table 3. Singular fiber types and the corresponding gauge groups on the discriminant components of a complete intersection (A.1).

We compute the Mordell-Weil group of the Jacobian fibration (A.18).

The projection from the Jacobian $(A .18)$ onto $\mathbb{P}^{1} \times \mathbb{P}^{1}$ (on which polynomials $f, g$ are defined) gives a K3 fibration, whose fiber is given by the following equation:

$$
\tau^{2}=-t^{2} \lambda^{4}+\left(t^{4}+1\right) \lambda^{2}-t^{2}
$$

The Mordell-Weil group of the K3 surface (A.19) was determined to be $\mathbb{Z}_{4} \times \mathbb{Z}_{4}$ in [35]. We consider a specialization from the Jacobian fibration (A.18) to its K3 fiber (A.19), which is equivalent to selecting a point in the base surface, $\mathbb{P}^{1} \times \mathbb{P}^{1}$, to deduce that the MordellWeil group of the Jacobian fibration (A.18) is isomorphic to that of the K3 surface (A.19). Therefore, we conclude that the Mordell-Weil group of the Jacobian fibration (A.18) is $\mathbb{Z}_{4} \times \mathbb{Z}_{4}$. Thus, the global structure of the non-Abelian gauge group is

$$
\mathrm{SU}(4)^{4} \times \mathrm{SU}(2)^{4} / \mathbb{Z}_{4} \times \mathbb{Z}_{4}
$$

In particular, the Mordell-Weil group of the Jacobian (A.18) has rank 0. Thus, Ftheory compactifications on the complete intersection Calabi-Yau 4-folds (A.1) do not have a $\mathrm{U}(1)$ gauge field.

\section{A.3 Euler characteristic and condition on 4-form flux to cancel the tadpole}

As stated earlier, we do not discuss whether a consistent flux exists. By computing the Euler characteristic of $(2,1,1,1)$ and $(2,1,1,1)$ complete intersection Calabi-Yau 4-folds in $\mathbb{P}^{3} \times \mathbb{P}^{1} \times \mathbb{P}^{1} \times \mathbb{P}^{1}$, we derive a condition imposed on the self-intersection of a 4 -form flux $G_{4}$ to cancel the tadpole.

We compute the Euler characteristic $\chi(Y)$ of a $(2,1,1,1)$ and $(2,1,1,1)$ complete intersection Calabi-Yau 4-fold $Y$. An exact sequence,

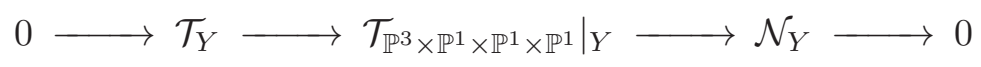

gives the following equality:

$$
c\left(\mathcal{T}_{Y}\right)=\frac{\left.c\left(\mathcal{T}_{\left.\mathbb{P}^{3} \times \mathbb{P}^{1} \times \mathbb{P}^{1} \times \mathbb{P}^{1}\right)}\right)\right|_{Y}}{c\left(\mathcal{N}_{Y}\right)} .
$$

In the sequence (A.21), $\mathcal{T}_{Y}$ is the tangent bundle of the $(2,1,1,1)$ and $(2,1,1,1)$ complete intersection Calabi-Yau 4-fold $Y$, and $\left.\right|_{Y}$ represents the restriction to $Y . \mathcal{N}_{Y}$ is the normal bundle resulting from natural embedding of tangent bundle $\mathcal{T}_{Y}$ into tangent bundle 


\begin{tabular}{|c|c|c|}
\hline CY 4-fold $Y$ & Euler char. $\chi(Y)$ & $\frac{\chi(Y)}{24}$ \\
\hline$(2,1,1,1)$ and $(2,1,1,1)$ complete intersection & 864 & 36 \\
\hline
\end{tabular}

Table 4. Euler characteristic of complete intersections of $(2,1,1,1)$ and $(2,1,1,1)$ hypersurfaces in $\mathbb{P}^{3} \times \mathbb{P}^{1} \times \mathbb{P}^{1} \times \mathbb{P}^{1}$.

$\mathcal{T}_{\mathbb{P}^{3} \times \mathbb{P}^{1} \times \mathbb{P}^{1} \times \mathbb{P}^{1}}$ of the ambient space, $\mathbb{P}^{3} \times \mathbb{P}^{1} \times \mathbb{P}^{1} \times \mathbb{P}^{1}$.

$$
\mathcal{N}_{Y} \cong \mathcal{O}(2,1,1,1)^{\oplus 2},
$$

thus,

$$
c\left(\mathcal{N}_{Y}\right)=(1+2 x+y+z+w)^{2} .
$$

We have

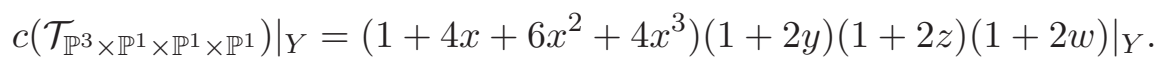

From equation (A.22), we find that the Euler characteristic $\chi(Y)$, which is equal to the top Chern class of $c(Y)$, is

$$
\chi(Y)=864
$$

This is divisible by 24 , and we have

$$
\frac{\chi(Y)}{24}=\frac{864}{24}=36
$$

Additionally, we find the second Chern class $c_{2}(Y)$ from equation (A.22) as follows:

$$
c_{2}(Y)=\left.\left(2 x^{2}+4 x y+4 x z+4 x w+2 y z+2 y w+2 z w\right)\right|_{Y} .
$$

This is even; thus, the quantization condition for a 4 -form flux $G_{4}$ [73] reduces to

$$
G_{4} \in H^{4}(Y, \mathbb{Z})
$$

A bound on the self-intersection of a 4 -form flux $G_{4}$ to cancel the tadpole is

$$
N_{3}=\frac{\chi(Y)}{24}-\frac{1}{2} G_{4} \cdot G_{4}=36-\frac{1}{2} G_{4} \cdot G_{4} \geq 0
$$

with $N_{3}$ the number of 3-branes minus anti 3-branes.

\section{A.4 Potential matter fields and Yukawa couplings}

We deduce the potential matter fields on 7-branes and along matter curves, and Yukawa couplings. As stated earlier, it is undetermined whether a consistent flux exists. Therefore, we can only say that the matter spectra (and Yukawa couplings) we compute could arise.

A deformation of the singularity associated with a gauge group $G$ generates matter fields on 7-branes [76, 77]. As stated in [78], this corresponds to the breaking of the gauge group $G$ on 7 -branes to a subgroup $\Gamma$, with maximal inclusion

$$
\Gamma \times H \subset G .
$$


We focus on the case in which $H$ is $\mathrm{U}(1)$.

We focus on the bulk component, $E_{1}$, of the discriminant locus. (Component $E_{1}$ is discussed in A.1.) We abbreviate $E_{1}$ to $E$. Let $\mathcal{L}$ be a supersymmetric line bundle on the bulk $E$. As $E \cong \mathbb{P}^{1} \times \mathbb{P}^{1}$ (see table 2 ), $\mathcal{L} \cong \mathcal{O}(a, b)$ for some integers $a, b \in \mathbb{Z}$. As argued in [78], integers $a, b$ are required to satisfy the following inequality:

$$
a b<0
$$

for the line bundle $\mathcal{L} \cong \mathcal{O}(a, b)$ to be supersymmetric.

Suppose $\Gamma$ has a representation $\tau$ with $\mathrm{U}(1)$ charge $n$. Then, the line bundle associated to a matter in the representation $\tau$ is $\mathcal{L}^{n}$. The generation of matters in the representation $\tau$ of $\Gamma$ on the bulk $E$ is given by the following equation [78]:

$$
n_{\tau}-n_{\tau^{*}}=-\int_{E} c_{1}(E) c_{1}\left(\mathcal{L}^{n}\right)=-n \int_{E} c_{1}(E) c_{1}(\mathcal{L})
$$

When the gauge group $\mathrm{SU}(4)$ on the bulk $E$ breaks to $\mathrm{SU}(3)$ under

$$
\mathrm{SU}(4) \supset \mathrm{SU}(3) \times \mathrm{U}(1)
$$

adjoint $\mathbf{1 5}$ of SU(4) decomposes as [79]

$$
\mathbf{1 5}=\mathbf{8}_{0}+\mathbf{3}_{-4}+\overline{\mathbf{3}}_{4}+\mathbf{1}_{0}
$$

Thus, chiral matters $\mathbf{3}$ (could) arise on the bulk $E$, and their generation is given by

$$
\begin{aligned}
n_{\mathbf{3}}-n_{\overline{\mathbf{3}}} & =-\int_{E} c_{1}(E) c_{1}\left(\mathcal{L}^{-4}\right)=4(2 x+2 y)(a x+b y) \\
& =8(a+b) .
\end{aligned}
$$

We compute the generation of matter fields localized along the matter curve $E \cap D_{i}$, $i=1,2$. We saw in A.1 that the matter curve $E \cap D_{i}, i=1,2$, is isomorphic to a rational curve $\Sigma_{0}, E \cap D_{i} \cong \Sigma_{0}, i=1,2$. (See equation (A.10) in A.1.) $\Sigma_{0}$ denotes a Riemann surface of genus 0 , namely, a rational curve $\mathbb{P}^{1}$.

$E \cap D_{i}, i=1,2$, is a bidegree $(1,1)$ curve in $\mathbb{P}^{1} \times \mathbb{P}^{1}$. Therefore, the restriction $\mathcal{L}_{\Sigma_{0}}$, of the line bundle $\mathcal{L} \cong \mathcal{O}(a, b)$ to the matter curve $E \cap D_{i}=\Sigma_{0}, i=1,2$, is

$$
\mathcal{L}_{\Sigma_{0}} \cong \mathcal{O}_{\Sigma_{0}}(a+b) \text {. }
$$

Matter field 6 localized along the matter curve $E \cap D_{i} \cong \Sigma_{0}, i=1,2$, decomposes as

$$
\mathbf{6}=\mathbf{3}_{2}+\overline{\mathbf{3}}_{-2} .
$$

We apply the Riemann-Roch theorem to obtain

$$
\begin{aligned}
n_{\mathbf{3}} & =h^{0}\left(K_{\Sigma_{0}}^{1 / 2} \otimes \mathcal{L}_{\Sigma_{0}}^{2}\right) \\
& =h^{0}\left(\mathcal{O}_{\Sigma_{0}}(2(a+b)-1)\right) \\
& =\left\{\begin{array}{rr}
2(a+b) & (a+b \geq 0) \\
0 & (a+b<0)
\end{array}\right.
\end{aligned}
$$




\begin{tabular}{|c|c|c|c|c|c|c|}
\hline Gauge Group & $a+b$ & Matters on $E$ & \# Gen. on $E$ & Matters on $\Sigma_{0}$ & \# Gen. on $\Sigma_{0}$ & Yukawa \\
\hline \multirow{2}{*}{$\mathrm{SU}(4)$} & $>0$ & $\mathbf{3}$ & $8(a+b)$ & $\mathbf{3}$ & $2(a+b)$ & $\mathbf{3} \cdot \mathbf{3} \cdot \mathbf{3}$ \\
\cline { 2 - 7 } & $<0$ & $\overline{\mathbf{3}}$ & $-8(a+b)$ & $\overline{\mathbf{3}}$ & $-2(a+b)$ & $\overline{\mathbf{3}} \cdot \overline{\mathbf{3}} \cdot \overline{\mathbf{3}}$ \\
\hline
\end{tabular}

Table 5. Potential matter spectra in F-theory compactification on $(2,1,1,1)$ and $(2,1,1,1)$ complete intersection (A.1) in $\mathbb{P}^{3} \times \mathbb{P}^{1} \times \mathbb{P}^{1} \times \mathbb{P}^{1}$.

Similarly,

$$
\begin{aligned}
n_{\overline{\mathbf{3}}} & =h^{0}\left(K_{\Sigma_{0}}^{1 / 2} \otimes \mathcal{L}_{\Sigma_{0}}^{-2}\right) \\
& =h^{0}\left(\mathcal{O}_{\Sigma_{0}}(-2(a+b)-1)\right) \\
& =\left\{\begin{array}{rr}
-2(a+b) & (a+b \leq 0) \\
0 & (a+b>0)
\end{array}\right.
\end{aligned}
$$

Thus, when $a+b>0,3$ (could) localize along the matter curve $E \cap D_{i} \cong \Sigma_{0}, i=1,2$. When $a+b<0$, matter fields $\overline{\mathbf{3}}$ (could) localize along the matter curve $E \cap D_{i} \cong \Sigma_{0}, i=1,2$.

As discussed in [78], Yukawa coupling arises from the interactions of the following three cases: i)Two matter fields on a matter curve and a matter on a bulk, ii)Three fields on a bulk, and iii)Matters along three matter curves intersecting at one point. $E \cong \mathbb{P}^{1} \times \mathbb{P}^{1}$ is a Hirzebruch surface; thus, Yukawa coupling does not arise from the interaction of the second case on the bulk $E$, as stated in [78]. We focus on Yukawa couplings arising from the first case.

When $a+b>0$, matter field $\mathbf{3}$ on the bulk $E$ and two matter fields $\mathbf{3}$ localized along the matter curve $\Sigma_{0}$ generate the following Yukawa coupling:

$$
3 \cdot 3 \cdot 3 \text {. }
$$

When $a+b<0$, matter field $\overline{\mathbf{3}}$ on the bulk $E$ and two matter fields $\overline{\mathbf{3}}$ localized along the matter curve $\Sigma_{0}$ generate the following Yukawa coupling:

$$
\overline{\mathbf{3}} \cdot \overline{\mathbf{3}} \cdot \overline{\mathbf{3}} \text {. }
$$

The results are displayed in table 5 .

We saw in section 3.5 that F-theory model on the Calabi-Yau complete intersection (A.1) has a discrete $\mathbb{Z}_{2}$ symmetry; therefore massless matter fields are charged under a discrete $\mathbb{Z}_{2}$ symmetry, and this has to be reflected in the structure of Yukawa couplings [28]. Yukawa couplings (A.41) and (A.42) are indeed invariant under the action of $\mathbb{Z}_{2}$.

Open Access. This article is distributed under the terms of the Creative Commons Attribution License (CC-BY 4.0), which permits any use, distribution and reproduction in any medium, provided the original author(s) and source are credited.

\section{References}

[1] C. Vafa, Evidence for F-theory, Nucl. Phys. B 469 (1996) 403 [hep-th/9602022] [INSPIRE]. 
[2] D.R. Morrison and C. Vafa, Compactifications of F-theory on Calabi-Yau threefolds. 1, Nucl. Phys. B 473 (1996) 74 [hep-th/9602114] [INSPIRE].

[3] D.R. Morrison and C. Vafa, Compactifications of F-theory on Calabi-Yau threefolds. 2, Nucl. Phys. B 476 (1996) 437 [hep-th/9603161] [InSPIRE].

[4] T.W. Grimm and T. Weigand, On Abelian gauge symmetries and proton decay in global F-theory GUTs, Phys. Rev. D 82 (2010) 086009 [arXiv: 1006.0226] [INSPIRE].

[5] D.R. Morrison and D.S. Park, F-theory and the Mordell-Weil group of elliptically-fibered Calabi-Yau threefolds, JHEP 10 (2012) 128 [arXiv:1208.2695] [INSPIRE].

[6] M. Cvetič, T.W. Grimm and D. Klevers, Anomaly cancellation and Abelian gauge symmetries in F-theory, JHEP 02 (2013) 101 [arXiv:1210.6034] [INSPIRE].

[7] C. Mayrhofer, E. Palti and T. Weigand, U(1) symmetries in F-theory GUTs with multiple sections, JHEP 03 (2013) 098 [arXiv:1211.6742] [INSPIRE].

[8] V. Braun, T.W. Grimm and J. Keitel, New global F-theory GUTs with U(1) symmetries, JHEP 09 (2013) 154 [arXiv:1302.1854] [INSPIRE].

[9] J. Borchmann, C. Mayrhofer, E. Palti and T. Weigand, Elliptic fibrations for $\mathrm{SU}(5) \times \mathrm{U}(1) \times \mathrm{U}(1)$ F-theory vacua, Phys. Rev. D 88 (2013) 046005 [arXiv: 1303.5054] [INSPIRE].

[10] M. Cvetič, D. Klevers and H. Piragua, F-theory compactifications with multiple U(1)-factors: constructing elliptic fibrations with rational sections, JHEP 06 (2013) 067 [arXiv: 1303.6970] [INSPIRE].

[11] V. Braun, T.W. Grimm and J. Keitel, Geometric engineering in toric F-theory and GUTs with U(1) gauge factors, JHEP 12 (2013) 069 [arXiv: 1306.0577] [INSPIRE].

[12] M. Cvetič, A. Grassi, D. Klevers and H. Piragua, Chiral four-dimensional F-theory compactifications with $\mathrm{SU}(5)$ and multiple $\mathrm{U}(1)$-factors, JHEP 04 (2014) 010 [arXiv: 1306.3987] [INSPIRE].

[13] J. Borchmann, C. Mayrhofer, E. Palti and T. Weigand, SU(5) tops with multiple U(1)s in F-theory, Nucl. Phys. B 882 (2014) 1 [arXiv:1307.2902] [InSPIRE].

[14] M. Cvetič, D. Klevers and H. Piragua, F-theory compactifications with multiple U(1)-factors: addendum, JHEP 12 (2013) 056 [arXiv:1307.6425] [INSPIRE].

[15] M. Cvetič, D. Klevers, H. Piragua and P. Song, Elliptic fibrations with rank three Mordell-Weil group: F-theory with $\mathrm{U}(1) \times \mathrm{U}(1) \times \mathrm{U}(1)$ gauge symmetry, JHEP 03 (2014) 021 [arXiv:1310.0463] [INSPIRE].

[16] M. Bies, C. Mayrhofer, C. Pehle and T. Weigand, Chow groups, Deligne cohomology and massless matter in F-theory, arXiv:1402.5144 [INSPIRE].

[17] T.W. Grimm and A. Kapfer, Anomaly cancelation in field theory and F-theory on a circle, JHEP 05 (2016) 102 [arXiv: 1502.05398] [INSPIRE].

[18] C. Lawrie, S. Schäfer-Nameki and J.-M. Wong, F-theory and all things rational: surveying $\mathrm{U}(1)$ symmetries with rational sections, JHEP 09 (2015) 144 [arXiv: 1504.05593] [INSPIRE].

[19] M. Cvetič, D. Klevers, H. Piragua and W. Taylor, General U(1) × U(1) F-theory compactifications and beyond: geometry of unHiggsings and novel matter structure, JHEP 11 (2015) 204 [arXiv: 1507.05954] [INSPIRE].

[20] M. Cvetič, A. Grassi, D. Klevers, M. Poretschkin and P. Song, Origin of Abelian gauge symmetries in heterotic/F-theory duality, JHEP 04 (2016) 041 [arXiv:1511.08208] [INSPIRE]. 
[21] D.R. Morrison and D.S. Park, Tall sections from non-minimal transformations, JHEP 10 (2016) 033 [arXiv: 1606.07444] [INSPIRE].

[22] V. Braun and D.R. Morrison, F-theory on genus-one fibrations, JHEP 08 (2014) 132 [arXiv: 1401.7844] [INSPIRE].

[23] D.R. Morrison and W. Taylor, Sections, multisections and U(1) fields in F-theory, arXiv: 1404.1527 [INSPIRE].

[24] P. Berglund, J.R. Ellis, A.E. Faraggi, D.V. Nanopoulos and Z. Qiu, Elevating the free fermion $Z_{2} \times Z_{2}$ orbifold model to a compactification of F-theory, Int. J. Mod. Phys. A 15 (2000) 1345 [hep-th/9812141] [InSPIRE].

[25] J. de Boer et al., Triples, fluxes and strings, Adv. Theor. Math. Phys. 4 (2002) 995 [hep-th/0103170] [INSPIRE].

[26] L.B. Anderson, I. García-Etxebarria, T.W. Grimm and J. Keitel, Physics of F-theory compactifications without section, JHEP 12 (2014) 156 [arXiv:1406.5180] [INSPIRE].

[27] D. Klevers, D.K. Mayorga Pena, P.-K. Oehlmann, H. Piragua and J. Reuter, F-theory on all toric hypersurface fibrations and its Higgs branches, JHEP 01 (2015) 142 [arXiv:1408.4808] [INSPIRE].

[28] I. García-Etxebarria, T.W. Grimm and J. Keitel, Yukawas and discrete symmetries in F-theory compactifications without section, JHEP 11 (2014) 125 [arXiv:1408.6448] [INSPIRE].

[29] C. Mayrhofer, E. Palti, O. Till and T. Weigand, Discrete gauge symmetries by Higgsing in four-dimensional F-theory compactifications, JHEP 12 (2014) 068 [arXiv:1408.6831] [INSPIRE].

[30] C. Mayrhofer, E. Palti, O. Till and T. Weigand, On discrete symmetries and torsion homology in F-theory, JHEP 06 (2015) 029 [arXiv: 1410.7814] [INSPIRE].

[31] M. Cvetič, R. Donagi, D. Klevers, H. Piragua and M. Poretschkin, F-theory vacua with $Z_{3}$ gauge symmetry, Nucl. Phys. B 898 (2015) 736 [arXiv:1502.06953] [INSPIRE].

[32] L. Lin, C. Mayrhofer, O. Till and T. Weigand, Fluxes in F-theory compactifications on genus-one fibrations, JHEP 01 (2016) 098 [arXiv:1508.00162] [INSPIRE].

[33] T.W. Grimm, A. Kapfer and D. Klevers, The arithmetic of elliptic fibrations in gauge theories on a circle, JHEP 06 (2016) 112 [arXiv:1510.04281] [INSPIRE].

[34] Y. Kimura, Gauge groups and matter fields on some models of F-theory without section, JHEP 03 (2016) 042 [arXiv:1511.06912] [INSPIRE].

[35] Y. Kimura, Gauge groups and matter fields for F-theory without section on double covers of $P^{1} \times P^{1}$ and complete intersections in $P^{1} \times P^{3}$, arXiv:1603.03212 [INSPIRE].

[36] Y. Kimura, F-theory models without section on Calabi-Yau 4-folds, arXiv:1607.02978 [INSPIRE].

[37] T. Kobayashi, H.P. Nilles, F. Ploger, S. Raby and M. Ratz, Stringy origin of non-Abelian discrete flavor symmetries, Nucl. Phys. B 768 (2007) 135 [hep-ph/0611020] [INSPIRE].

[38] H. Abe, K.-S. Choi, T. Kobayashi and H. Ohki, Non-Abelian discrete flavor symmetries from magnetized/intersecting brane models, Nucl. Phys. B 820 (2009) 317 [arXiv:0904.2631] [INSPIRE].

[39] T. Banks and N. Seiberg, Symmetries and strings in field theory and gravity, Phys. Rev. D 83 (2011) 084019 [arXiv:1011.5120] [InSPIRE]. 
[40] S. Hellerman and E. Sharpe, Sums over topological sectors and quantization of Fayet-Iliopoulos parameters, Adv. Theor. Math. Phys. 15 (2011) 1141 [arXiv:1012.5999] [INSPIRE].

[41] P.G. Camara, L.E. Ibáñez and F. Marchesano, RR photons, JHEP 09 (2011) 110 [arXiv: 1106.0060] [INSPIRE].

[42] M. Berasaluce-Gonzalez, L.E. Ibáñez, P. Soler and A.M. Uranga, Discrete gauge symmetries in D-brane models, JHEP 12 (2011) 113 [arXiv:1106.4169] [INSPIRE].

[43] L.E. Ibáñez, A.N. Schellekens and A.M. Uranga, Discrete gauge symmetries in discrete MSSM-like orientifolds, Nucl. Phys. B 865 (2012) 509 [arXiv:1205.5364] [InSPIRE].

[44] M. Berasaluce-Gonzalez, P.G. Camara, F. Marchesano, D. Regalado and A.M. Uranga, Non-Abelian discrete gauge symmetries in 4d string models, JHEP 09 (2012) 059 [arXiv: 1206.2383] [INSPIRE].

[45] M. Berasaluce-Gonzalez, P.G. Camara, F. Marchesano and A.M. Uranga, $Z_{p}$ charged branes in flux compactifications, JHEP 04 (2013) 138 [arXiv:1211.5317] [INSPIRE].

[46] F. Marchesano, D. Regalado and L. Vazquez-Mercado, Discrete flavor symmetries in D-brane models, JHEP 09 (2013) 028 [arXiv: 1306.1284] [INSPIRE].

[47] G. Honecker and W. Staessens, To tilt or not to tilt: discrete gauge symmetries in global intersecting D-brane models, JHEP 10 (2013) 146 [arXiv:1303.4415] [INSPIRE].

[48] M. Berasaluce-González, G. Ramírez and A.M. Uranga, Antisymmetric tensor $Z_{p}$ gauge symmetries in field theory and string theory, JHEP 01 (2014) 059 [arXiv:1310.5582] [INSPIRE].

[49] A. Karozas, S.F. King, G.K. Leontaris and A. Meadowcroft, Discrete family symmetry from F-theory GUTs, JHEP 09 (2014) 107 [arXiv: 1406.6290] [InSPIRE].

[50] V. Braun, T.W. Grimm and J. Keitel, Complete intersection fibers in F-theory, JHEP 03 (2015) 125 [arXiv:1411.2615] [INSPIRE].

[51] G. Honecker and W. Staessens, Discrete Abelian gauge symmetries and axions, J. Phys. Conf. Ser. 631 (2015) 012080 [arXiv:1502.00985] [InSPIRE].

[52] T.W. Grimm, T.G. Pugh and D. Regalado, Non-Abelian discrete gauge symmetries in F-theory, JHEP 02 (2016) 066 [arXiv:1504.06272] [INSPIRE].

[53] M. Cvetič, A. Grassi and M. Poretschkin, Discrete symmetries in heterotic/F-theory duality and mirror symmetry, arXiv:1607.03176 [INSPIRE].

[54] V.A. Iskovskih, Fano 3-folds. I, Math. USSR Izv. 11 (1977) 485.

[55] T. Fujita, On the structure of polarized manifolds with total deficiency one, I, J. Math. Soc. Japan 32 (1980) 709.

[56] T. Fujita, On the structure of polarized manifolds with total deficiency one, II, J. Math. Soc. Japan 33 (1981) 415.

[57] K. Kodaira, On compact analytic surfaces II, Ann. Math. 77 (1963) 563.

[58] K. Kodaira, On compact analytic surfaces III, Ann. Math. 78 (1963) 1.

[59] A. Néron, Modèles minimaux des variétés abéliennes sur les corps locaux et globaux (in French), Publ. Math. IHÉS 21 (1964) 5.

[60] P.S. Aspinwall and D.R. Morrison, Nonsimply connected gauge groups and rational points on elliptic curves, JHEP 07 (1998) 012 [hep-th/9805206] [INSPIRE]. 
[61] C. Mayrhofer, D.R. Morrison, O. Till and T. Weigand, Mordell-Weil torsion and the global structure of gauge groups in F-theory, JHEP 10 (2014) 016 [arXiv:1405.3656] [INSPIRE].

[62] S. Gukov, C. Vafa and E. Witten, CFT's from Calabi-Yau four folds, Nucl. Phys. B 584 (2000) 69 [Erratum ibid. B 608 (2001) 477] [hep-th/9906070] [InSPIRE].

[63] A.P. Braun, A. Collinucci and R. Valandro, G-flux in F-theory and algebraic cycles, Nucl. Phys. B 856 (2012) 129 [arXiv:1107.5337] [INSPIRE].

[64] J. Marsano and S. Schäfer-Nameki, Yukawas, G-flux and spectral covers from resolved Calabi-Yau's, JHEP 11 (2011) 098 [arXiv:1108.1794] [INSPIRE].

[65] S. Krause, C. Mayrhofer and T. Weigand, $G_{4}$ flux, chiral matter and singularity resolution in F-theory compactifications, Nucl. Phys. B 858 (2012) 1 [arXiv:1109.3454] [INSPIRE].

[66] S. Krause, C. Mayrhofer and T. Weigand, Gauge fluxes in F-theory and type IIB orientifolds, JHEP 08 (2012) 119 [arXiv:1202.3138] [INSPIRE].

[67] N. Cabo Bizet, A. Klemm and D. Vieira Lopes, Landscaping with fluxes and the $E_{8}$ Yukawa point in F-theory, arXiv: 1404.7645 [INSPIRE].

[68] M. Cvetič, D. Klevers, D.K.M. Peña, P.-K. Oehlmann and J. Reuter, Three-family particle physics models from global F-theory compactifications, JHEP 08 (2015) 087 [arXiv: 1503.02068] [INSPIRE].

[69] S. Schäfer-Nameki and T. Weigand, F-theory and 2d (0,2) theories, JHEP 05 (2016) 059 [arXiv: 1601.02015] [INSPIRE].

[70] L. Lin and T. Weigand, $G_{4}$-flux and standard model vacua in F-theory, Nucl. Phys. B 913 (2016) 209 [arXiv:1604.04292] [inSPIRE].

[71] K. Becker and M. Becker, M theory on eight manifolds, Nucl. Phys. B 477 (1996) 155 [hep-th/9605053] [INSPIRE].

[72] S. Sethi, C. Vafa and E. Witten, Constraints on low dimensional string compactifications, Nucl. Phys. B 480 (1996) 213 [hep-th/9606122] [INSPIRE].

[73] E. Witten, On flux quantization in M-theory and the effective action, J. Geom. Phys. 22 (1997) 1 [hep-th/9609122] [INSPIRE].

[74] K. Dasgupta, G. Rajesh and S. Sethi, $M$ theory, orientifolds and $G$-flux, JHEP 08 (1999) 023 [hep-th/9908088] [INSPIRE].

[75] M. Schütt and T. Shioda, Elliptic surfaces, in Algebraic Geometry in East Asia, Seoul South Korea, (2008), Adv. Stud. Pure Math. 60 (2010) 51 [arXiv:0907.0298].

[76] M. Bershadsky, K.A. Intriligator, S. Kachru, D.R. Morrison, V. Sadov and C. Vafa, Geometric singularities and enhanced gauge symmetries, Nucl. Phys. B 481 (1996) 215 [hep-th/9605200] [INSPIRE].

[77] S.H. Katz and C. Vafa, Matter from geometry, Nucl. Phys. B 497 (1997) 146 [hep-th/9606086] [INSPIRE].

[78] C. Beasley, J.J. Heckman and C. Vafa, GUTs and exceptional branes in F-theory - I, JHEP 01 (2009) 058 [arXiv:0802.3391] [INSPIRE].

[79] R. Slansky, Group theory for unified model building, Phys. Rept. 79 (1981) 1 [INSPIRE]. 